\title{
Attribute Reduction in Intuitionistic Fuzzy Concept Lattices
}

\author{
Jinzhong Pang, Xiaoyan Zhang, and Weihua Xu \\ School of Mathematics and Statistics, Chongqing University of Technology, Chongqing 400054, China \\ Correspondence should be addressed to Xiaoyan Zhang; zhangxyms@gmail.com
}

Received 29 March 2013; Revised 24 June 2013; Accepted 15 August 2013

Academic Editor: Jose L. Gracia

Copyright (C) 2013 Jinzhong Pang et al. This is an open access article distributed under the Creative Commons Attribution License, which permits unrestricted use, distribution, and reproduction in any medium, provided the original work is properly cited.

\begin{abstract}
As an effective tool for knowledge discovery, concept lattice has been successfully applied to various fields. And one of the key problems of knowledge discovery is attribute reduction. In order to understand the problems better, the attribute reduction is necessary to perfect the theory as well as expand application of concept lattice. This paper introduces the intuitionistic fuzzy theory into the concept lattice theory and proposes a kind of intuitionistic fuzzy concept lattice. Then, an approach to attribute reduction based on the discernibility matrix is proposed and investigated, which makes the discovery of implicit knowledge easier and the representation simpler in data; furthermore, the theory of concept lattice is perfected. The theory of intuitionistic fuzzy concept lattice is useful and meaningful in view of the complexity and fuzziness of information in real world, and the potential value of dealing with information is expected in the future.
\end{abstract}

\section{Introduction}

Concept lattice stems from the so-called formal concept analysis proposed by Wille in 1982 [1], which can be depicted by a Hasse diagram, where each node expresses a formal concept. A concept lattice is an ordered hierarchical structure of formal concepts that are defined by a binary relation between a set of objects and a set of attributes. Concretely, each formal concept is the pair (objects, attributes), which consists of two parts: the extension (objects covered by the concept) and intension (attributes describing the concept). As an effective tool for data analysis and knowledge processing, concept lattice has been applied to various fields, such as data mining, information retrieval, and software engineering [24].

The intuitionistic fuzzy (IF, for short) set theory initiated by Atanassov $[5,6]$ is also an important mathematical structure to cope with imprecise information. IF set, as an extension of Zadeh's fuzzy set [7], considers both membership degree and nonmembership degree which are functions valued in $[0,1]$, while a fuzzy set gives a membership degree only. The membership and nonmembership values induce an indeterminacy index, which models the hesitancy degree of how an object satisfies a particular property. So IF set theory can present vague information better. Recently, IF set theory has been successfully applied in decision analysis and pattern recognition [8-11].

In recent years, many new achievements on these topics have been achieved on theories such as construction of concept lattice $[1,2,12-15]$ and acquisition of rules [16, 17]. For example, the paper [15] shows that an approach of creating fuzzy concept lattices proposed by Popescu was equivalent to the approach of Krajči called generalized concept lattices in some way by comparing this approach with several other approaches and give a straightforward generalization of Popescu's approach to nonhomogeneous cases, and Li et al. [17] investigate the issue of rule acquisition in incomplete decision context. And although rough set theory and formal context analysis are different theories, they have much in common in terms of both goals and methodologies, and a formal context in formal concept analysis corresponds to an information system in rough set theory $[18,19]$. Formal concept analysis abstracts the knowledge from a formal context through formal concepts, while rough set theory discovers the knowledge via lower and upper approximations, positive boundary, and negative regions from an information system $[18,20,21]$. In fact, there are strong connections between formal concept analysis and rough set theory, and some researchers have been devoted to comparing and combining these two useful theories $[18,22-24]$, based on which we 
can study the concept lattice in the similar way to the rough set. Similarly, the key to attribute reduction is to find the minimal subsets of attributes sets in concept lattice, which can determine a concept lattice isomorphic to the one determined by all attributes while the object set remains unchanged. It makes the discovery of implicit knowledge easier and the representation simpler in data and extends the theory of concept lattice; knowledge reduction in formal concept analysis has attracted much attention [16, 17, 25-40]. For instance, Ganter and Wille [27] develop a reduction method to remove the reducible attributes and objects of a formal context via some predefined arrow relations. The paper [25] proposed a method to reduce the size of the concept lattice of a formal context using $K$-means clustering. Reduction approaches were presented to avoid the redundancy in the attributes from the perspectives of extension equivalence in the paper [39]. The paper [30] investigated the issue of developing efficient knowledge reduction methods for real decision formal contexts and developed a corresponding heuristic algorithm to search for a minimal reduction.

Similarly, attribute reduction in IF concept lattice is to find the minimum attribute set which can assign the same concepts and hierarchy based on IF formal context keeping the same objects. Reduction approaches in both the papers $[23,36]$ are based on the equivalence relation between the objects and attributes. In most situations, however, it is fuzzy or intuitionistic fuzzy. At present, there are some achievements on knowledge reduction based on fuzzy formal context $[15,25,28,34]$, where Lifeng Li introduced and investigated the attribute reduction in fuzzy concept lattices based on the kind of transitive implication operator. However, the study on attribute reduction for intuitionistic fuzzy formal context has not been investigated perfectly, although the discernibility matrix was proposed by using of the cuts of IF sets and then established the method of the attribute reduction of IF concept lattice in paper [24]. Because it leaves out some useful information about transforming the concept lattice based on the IF the formal context to a classical one only by using the cuts of the IF sets. Thus, for the requirement of knowledge-handling systems, combining IF set theory and formal concept analysis theory directly can result in a new hybrid mathematical structure by establishing two appropriate operators from other views.

Actually, the IF relation is an important type of data tables in formal concept analysis in real life. The paper combines the IF theory with the formal concept analysis, the main purpose of which is to study attribute reduction in IF concept lattices by introducing a pair of implication operators, and we establish approaches and theories of attribute reduction based on IF formal context, which is also suitable for classical formal context and fuzzy formal context.

The paper is organized as follows. Section 2 reviews basic definitions in formal concept analysis. We give the definitions and propositions in concept lattice with IF attributes in Section 3. In the next section, we discuss the corresponding definitions of attribute reduction in concept lattices, and then we divide the attributes into four types and investigate some related propositions and establish some propositions to determine the type of an attribute. In Section 5, the discernibility matrix and discernibility function in concept lattice are introduced, and then we discuss the approach to reduction as well as the corresponding characteristics. Finally, a simple conclusion is given in the paper.

\section{Preliminaries}

To make this paper self-contained, the IF set theory and involved notions of formal concept analysis are introduced briefly. Detailed description of them can be found in corresponding references.

Definition 1 (Ganter and Wille [27]). A triple $(U, A, I)$ is called a formal context, if $U=\left\{x_{1}, x_{2}, \ldots, x_{n}\right\}$ is an object set, where $x_{i}(i \leq n)$ is called an object; $A=\left\{a_{1}, a_{2}, \ldots, a_{m}\right\}$ is an attribute set, where $a_{j}(j \leq m)$ is called an attribute; and $I \subseteq U \times A$ is a binary relation between $U$ and $A$.

In a formal context $(U, A, I)$, if $(x, a) \in I$, that is, $x I a$, we say that the object $x$ has the attribute $a$, or that $a$ is fulfilled by $x$. For convenience, we use " 1 " and " 0 " to represent $(x, a) \in I$ and $(x, a) \notin I$, respectively. Thus, a formal context can be represented by a table only with 0 and 1 .

For a formal context $(U, A, I)$, a pair of dual operators for $X \subseteq U$ and $B \subseteq A$ is defined as follows:

$$
\begin{aligned}
& X^{*}=\{a \in A \mid(x, a) \in I, \forall x \in X\}, \\
& B^{*}=\{x \in U \mid(x, a) \in I, \forall a \in B\} .
\end{aligned}
$$

In fact, $X^{*}$ is the set of all the attributes shared by all the objects in $X$, and $B^{*}$ is the set of all the objects that fulfill all the attributes in $B$.

Meanwhile, the complement sets of $X^{*}$ and $B^{*}$ are denoted by $\sim X^{*}$ and $\sim B^{*}$, where $\sim X^{*}=\{a \in A \mid(x, a) \notin$ $I, \exists x \in X\}$ and $\sim B^{*}=\{x \in U \mid(x, a) \notin I, \exists a \in B\}$.

Proposition 2 (Ganter and Wille [27]). Let $(U, A, I)$ be a formal context, $X_{1}, X_{2}, X \subseteq U$ and $B_{1}, B_{2}, B \subseteq A$; the following properties hold:

(1) $X_{1} \subseteq X_{2} \Rightarrow X_{2}^{*} \subseteq X_{1}^{*}, B_{1} \subseteq B_{2} \Rightarrow B_{2}^{*} \subseteq B_{1}^{*}$.

(2) $X \subseteq X^{* *}, B \subseteq B^{* *}$.

(3) $X^{*}=X^{* * *} ; B^{*}=B^{* * *}$.

(4) $X \subseteq B^{*} \Leftrightarrow B \subseteq X^{*}$.

(5) $\left(X_{1} \cup X_{2}\right)^{*}=X_{1}^{*} \cap X_{2}^{*},\left(B_{1} \cup B_{2}\right)^{*}=B_{1}^{*} \cap B_{2}^{*}$.

(6) $\left(X_{1} \cap X_{2}\right)^{*} \supseteq X_{1}^{*} \cup X_{2}^{*},\left(B_{1} \cap B_{2}\right)^{*} \supseteq B_{1}^{*} \cup B_{2}^{*}$.

Definition 3 (Ganter and Wille [27]). Let $(U, A, I)$ be a formal context. A pair $(X, B)$ is called a formal concept (in brief a concept) if $X^{*}=B$ and $X=B^{*}$ for $X \subseteq U, B \subseteq$ $A$. Furthermore, $X$ and $B$ are called the extension and the intension of $(X, B)$, respectively.

From the above discussions, it is clear that both $\left(X^{* *}, X^{*}\right)$ and $\left(B^{*}, B^{* *}\right)$ are concepts.

According to $[23,27]$, we have the corresponding account as follows.

For convenience, all concepts of a formal context $(U, A, I)$ are denoted by $L(U, A, I)$, and they are ordered by

$$
\left(X_{1}, B_{1}\right) \leq\left(X_{2}, B_{2}\right) \Longleftrightarrow X_{1} \subseteq X_{2} \Longleftrightarrow B_{1} \supseteq B_{2},
$$


where $\left(X_{1}, B_{1}\right)$ and $\left(X_{2}, B_{2}\right)$ are concepts. Moreover, $\left(X_{1}, B_{1}\right)$ is called a subconcept of $\left(X_{2}, B_{2}\right)$, and $\left(X_{2}, B_{2}\right)$ is called a superconcept of $\left(X_{1}, B_{1}\right)$. And $\left(X_{1}, B_{1}\right)<\left(X_{2}, B_{2}\right)$ means that $\left(X_{1}, B_{1}\right) \leq\left(X_{2}, B_{2}\right)$ and $\left(X_{1}, B_{1}\right) \neq\left(X_{2}, B_{2}\right)$ hold at the same time. If $\left(X_{1}, B_{1}\right)<\left(X_{2}, B_{2}\right)$ and there does not exist a concept $(Y, C)$ such that $\left(X_{1}, B_{1}\right)<(Y, C)<\left(X_{2}, B_{2}\right)$, then $\left(X_{1}, B_{1}\right)$ is called a child concept (immediate subconcept) of $\left(X_{2}, B_{2}\right)$ and $\left(X_{2}, B_{2}\right)$ is called a parent concept (immediate superconcept $)$ of $\left(X_{1}, B_{1}\right)$, and this is denoted by $\left(X_{1}, B_{1}\right) \prec$ $\left(X_{2}, B_{2}\right)$.

For any two concepts $\left(X_{1}, B_{1}\right)$ and $\left(X_{2}, B_{2}\right)$ of a formal context $(U, A, I)$, it is easy to prove that both $\left(X_{1} \cap X_{2},\left(B_{1} \cup\right.\right.$ $\left.\left.B_{2}\right)^{* *}\right)$ and $\left(\left(X_{1} \cup X_{2}\right)^{* *}, B_{1} \cap B_{2}\right)$ are also concepts. Hence, if the meet and join are given by (Ganter and Wille [27]):

$$
\begin{aligned}
& \left(X_{1}, B_{1}\right) \wedge\left(X_{2}, B_{2}\right)=\left(X_{1} \cap X_{2},\left(B_{1} \cup B_{2}\right)^{* *}\right), \\
& \left(X_{1}, B_{1}\right) \vee\left(X_{2}, B_{2}\right)=\left(\left(X_{1} \cup X_{2}\right)^{* *}, B_{1} \cap B_{2}\right),
\end{aligned}
$$

then the concept lattice $L(U, A, I)$ is complete lattice.

Definition 4 (Atanassov [5]). Let $U$ be a finite and non-empty set called universe. An IF set $\widetilde{A}$ of $U$ has the following form:

$$
\widetilde{A}=\left\{\left\langle x, \mu_{\widetilde{A}}(x), \gamma_{\widetilde{A}}(x)\right\rangle \mid x \in U\right\},
$$

where $\mu_{\widetilde{A}}: U \rightarrow[0,1]$ and $\gamma_{\widetilde{A}}: U \rightarrow[0,1]$ and $\mu_{\widetilde{A}}(x)$ and $\gamma_{\widetilde{A}}(x)$ are, respectively, called the membership degree and nonmembership degree to $A$ of the object $x \in U$. Furthermore, they satisfy $0 \leq \mu_{\widetilde{A}}(x)+\gamma_{\widetilde{A}}(x) \leq 1$ for any $x \in U$. In general, we use $\mathscr{I} \mathscr{F}(U)$ to denote all IF sets in the universe $U$.

Definition 5 (Atanassov [6]). Let $\widetilde{A}, \widetilde{B} \in \mathscr{I} \mathscr{F}(U), \widetilde{A} \subseteq \widetilde{B} \Leftrightarrow$ $\mu_{\widetilde{A}}(x) \leq \mu_{\widetilde{B}}(x)$, and $\gamma_{\widetilde{A}}(x) \geq \gamma_{\widetilde{B}}(x)$ for any $x \in U$. If both $\widetilde{A} \subseteq \widetilde{B}$ and $\widetilde{B} \subseteq \widetilde{A}$, then we say $\widetilde{A}$ is equal to $\widetilde{B}$, denoted by $\widetilde{A}=\widetilde{B}$. The universe set and empty set are special IF set, where $\widetilde{U}=\{\langle x, 1,0\rangle \mid x \in U\}$ and $\widetilde{\emptyset}=\{\langle x, 0,1\rangle \mid x \in U\}$.

Let us denote intersection and union of $\widetilde{A}$ and $\widetilde{B}$ by $\widetilde{A} \cap \widetilde{B}$ and $\widetilde{A} \cup \widetilde{B}$, respectively. Moreover, we denote complement of $\widetilde{A}$ by $\sim \widetilde{A}$.

Definition 6 (Atanassov [6]). Let $\widetilde{A}, \widetilde{B} \in \mathscr{I} \mathscr{F}(U)$; then

$$
\begin{gathered}
\widetilde{A} \cap \widetilde{B}=\left\{\left\langle x, \wedge\left\{\mu_{\widetilde{A}}(x), \mu_{\widetilde{B}}(x)\right\}, \vee\left\{\gamma_{\widetilde{A}}(x), \gamma_{\widetilde{B}}(x)\right\}\right\rangle \mid x \in U\right\}, \\
\widetilde{A} \cup \widetilde{B}=\left\{\left\langle x, \vee\left\{\mu_{\widetilde{A}}(x), \mu_{\widetilde{B}}(x)\right\}, \wedge\left\{\gamma_{\widetilde{A}}(x), \gamma_{\widetilde{B}}(x)\right\}\right\rangle \mid x \in U\right\}, \\
\sim \widetilde{A}=\left\{\left\langle x, \gamma_{\widetilde{A}}(x), \mu_{\widetilde{A}}(x)\right\rangle \mid x \in U\right\} .
\end{gathered}
$$

Many properties of these operators in IF set theory are similar to fuzzy set theory. Detailed description can be found easily in the corresponding references.

\section{IF Concept Lattice}

The definition of concept lattice with IF attributes is introduced, and some important properties are discussed in this section.
TABLE 1

\begin{tabular}{cccccc}
\hline & $a$ & $b$ & $c$ & $d$ & $e$ \\
\hline$x_{1}$ & $\langle 0.9,0.0\rangle$ & $\langle 0.7,0.2\rangle$ & $\langle 0.2,0.5\rangle$ & $\langle 0.9,0.1\rangle$ & $\langle 0.8,0.1\rangle$ \\
$x_{2}$ & $\langle 0.8,0.1\rangle$ & $\langle 0.8,0.2\rangle$ & $\langle 0.8,0.1\rangle$ & $\langle 0.3,0.5\rangle$ & $\langle 0.2,0.7\rangle$ \\
$x_{3}$ & $\langle 0.1,0.8\rangle$ & $\langle 0.2,0.6\rangle$ & $\langle 0.1,0.9\rangle$ & $\langle 0.8,0.2\rangle$ & $\langle 0.2,0.6\rangle$ \\
$x_{4}$ & $\langle 0.7,0.2\rangle$ & $\langle 0.8,0.1\rangle$ & $\langle 0.7,0.1\rangle$ & $\langle 0.2,0.6\rangle$ & $\langle 0.2,0.8\rangle$ \\
\hline
\end{tabular}

Definition 7. A triple $(U, A, \widetilde{I})$ is called an IF formal context, if $U=\left\{x_{1}, x_{2}, \ldots, x_{n}\right\}$ is an object set, where $x_{i}(i \leq n)$ is called an object; $A=\left\{a_{1}, a_{2}, \ldots, a_{m}\right\}$ is an attribute set, where $a_{j}(j \leq m)$ is called an attribute; and $\widetilde{I}$ is an IF set of $U \times A$, where $\widetilde{I}=\left\{\left\langle(x, a), \mu_{\widetilde{I}}(x, a), \gamma_{\widetilde{I}}(x, a)\right\rangle \mid(x, a) \in U \times A\right\}, \mu_{\widetilde{I}}$ : $U \times A \rightarrow[0,1]$, and $\gamma_{\tilde{I}}: U \times A \rightarrow[0,1]$.

The complement of $\widetilde{I}$ is denoted by $\sim \widetilde{I}=$ $\left\{\left\langle(x, a), \gamma_{\widetilde{I}}(x, a), \mu_{\tilde{I}}(x, a)\right\rangle \mid(x, a) \in U \times A\right\}$.

We denote $\widetilde{I}(x, a)=\left\langle\mu_{\widetilde{I}}(x, a), \gamma_{\widetilde{I}}(x, a)\right\rangle$; then the set of $\widetilde{I}(x, a)(x \in U, a \in A)$ is denoted by

$$
V=\{\widetilde{I}(x, a) \mid x \in U, a \in A\} .
$$

Let $\widetilde{I}(x, a), \widetilde{I}(y, a) \in V$; then

$$
\begin{aligned}
\widetilde{I}(x, a) & \geq \widetilde{I}(y, a) \Longleftrightarrow \mu_{\widetilde{I}}(x, a) \\
& \geq \mu_{\widetilde{I}}(y, a) \wedge \gamma_{\widetilde{I}}(x, a) \leq \gamma_{\widetilde{I}}(y, a) .
\end{aligned}
$$

With respect to an IF formal context $(U, A, \widetilde{I})$, for $X \subseteq U$, $B \subseteq A$ and $\widetilde{A}, \widetilde{B} \in \mathscr{I} \mathscr{F}(U)$, where $\forall \widetilde{B}(b), \widetilde{A}(b) \in\{\widetilde{I}(x, b) \mid$ $\forall x \in U\}$.

A pair of operators is defined by

$$
X^{*}=\widetilde{A}=\left\{\left\langle a, \mu_{X^{*}}(a), \gamma_{X^{*}}(a)\right\rangle \mid a \in A\right\},
$$

where $\widetilde{A}(a)=\left\langle\wedge_{\forall x \in X} \mu_{\widetilde{I}}(x, a), \vee_{\forall x \in X} \gamma_{\widetilde{I}}(x, a)\right\rangle(a \in A)$ and denote $\emptyset^{*}=\widetilde{A}=\{\langle a, 1,0\rangle \mid a \in A\}$.

$$
\widetilde{B}^{*}=\{x \in U \mid \widetilde{I}(x, b) \geq \widetilde{B}(b), \forall b \in B\},
$$

where $\widetilde{I}(x, b) \in V$ and denote $\widetilde{B}(b)=\langle 0,1\rangle$, if $b \notin B$.

Similarly, $\forall x \in U$, we use $x^{*}$ and $a^{*}$ instead of $\{x\}^{*}$ and $\{a\}^{*}$, respectively, and for any $B \subseteq A$ denote

$$
U^{B}=\{\widetilde{B} \mid \widetilde{B}(b)=\widetilde{I}(x, b), x \in U, b \in B\} .
$$

Example 8. An IF formal context is shown as in Table 1.

In this context, let $X=\left\{x_{1}, x_{2}, x_{4}\right\}$ and $\widetilde{B}=$ $\{(a, 0.7,0.3),(b, 0.8,0.2),(c, 0.7,0.1),(d, 0.1,0.8)\} \in \mathscr{I} \mathscr{F}(B)$, where $B=\{a, b, c, d\} \subset A$; then from the definition we can obtain that

$$
\begin{gathered}
X^{*}=\widetilde{A}=\{(a, 0.7,0.2),(b, 0.7,0.2),(c, 0.2,0.5), \\
(d, 0.2,0.6),(e, 0.2,0.8)\}, \\
\widetilde{B}^{*}=\left\{x_{2}, x_{4}\right\} .
\end{gathered}
$$


Proposition 9. Let $(U, A, \widetilde{I})$ be an IF formal context, $X_{1}, X_{2}, X \subseteq U, B_{1}, B_{2}, B \subseteq A$; then the above operators have the following properties:

(1) $X_{1} \subseteq X_{2} \Rightarrow X_{2}^{*} \subseteq X_{1}^{*}, \widetilde{B}_{1} \subseteq \widetilde{B}_{2} \Rightarrow \widetilde{B}_{2}^{*} \subseteq \widetilde{B}_{1}^{*}$.

(2) $X \subseteq X^{* *}, \widetilde{B} \subseteq \widetilde{B}^{* *}$.

(3) $X^{*}=X^{* * *}, \widetilde{B}^{*}=\widetilde{B}^{* * *}$.

(4) $X \subseteq \widetilde{B}^{*} \Leftrightarrow \widetilde{B} \subseteq X^{*}$.

(5) $\left(X_{1} \cup X_{2}\right)^{*}=X_{1}^{*} \cap X_{2}^{*},\left(\widetilde{B}_{1} \cup \widetilde{B}_{2}\right)^{*}=\widetilde{B}_{1}^{*} \cap \widetilde{B}_{2}^{*}$.

(6) $\left(X_{1} \cap X_{2}\right)^{*} \supseteq X_{1}^{*} \cup X_{2}^{*},\left(\widetilde{B}_{1} \cap \widetilde{B}_{2}\right)^{*} \supseteq \widetilde{B}_{1}^{*} \cup \widetilde{B}_{2}^{*}$.

Proof.

(1) Denote that $X_{1}^{*}=\widetilde{A}_{1}$ and $X_{2}^{*}=\widetilde{A}_{2}$, so it can be known for any $a \in A$

$$
\begin{aligned}
& \widetilde{A}_{1}(a)=\left\langle\underset{\forall x \in X_{1}}{\wedge} \mu_{\widetilde{I}}(x, a), \underset{\forall x \in X_{1}}{\vee} \gamma_{\widetilde{I}}(x, a)\right\rangle, \\
& \widetilde{A}_{2}(a)=\left\langle\underset{\forall x \in X_{2}}{\wedge} \mu_{\widetilde{I}}(x, a), \underset{\forall x \in X_{2}}{\vee} \gamma_{\widetilde{I}}(x, a)\right\rangle,
\end{aligned}
$$

Since $X_{1} \subseteq X_{2}$, it is true that $\widetilde{A_{1}}(a) \geq \widetilde{A_{2}}(a)$. It follows that $\widetilde{A}_{2} \subseteq \widetilde{A}_{1}$; that is, $X_{2}^{*} \subseteq X_{1}^{*}$.

In addition, from the above definitions, we can obtain that if $a \in B_{i}(i=1,2), \widetilde{B}_{i}(a)=\widetilde{A}(a)$, otherwise $\widetilde{B}_{i}(a)=\langle 0,1\rangle$. So

$\widetilde{B}_{1}^{*}=\left\{x \in U \mid \widetilde{I}(x, b) \geq \widetilde{B}_{1}(b), \forall b \in A\right\}, \quad$ where $\widetilde{I}(x, b) \in V$, $\widetilde{B}_{2}^{*}=\left\{x \in U \mid \widetilde{I}(x, b) \geq \widetilde{B}_{2}(b), \forall b \in A\right\}, \quad$ where $\widetilde{I}(x, b) \in V$.

Since $\widetilde{B}_{1} \subseteq \widetilde{B}_{2}$, we can obtain that $\widetilde{B}_{1}(b) \leq \widetilde{B}_{2}(b)$. It follows that $\widetilde{I}(x, b) \geq \widetilde{B}_{2}(b)$ implies $\widetilde{I}(x, b) \geq \widetilde{B}_{1}(b)$ for any $\widetilde{I}(x, b) \in V$. Hence, if $x \in \widetilde{B}_{2}^{*}$, then $x \in \widetilde{B}_{1}^{*}$; that is, $\widetilde{B}_{2}^{*} \subseteq \widetilde{B}_{1}^{*}$.

(2) On one hand, assume that $X^{*}=\widetilde{A}$; then $X^{* *}=\widetilde{A}^{*}=$ $\{x \in U \mid \widetilde{I}(x, a) \geq \widetilde{A}(a), \forall a \in A\}$, where $\widetilde{A}(a)=$ $\wedge\{\widetilde{I}(x, a) \forall x \in X\}=\left\langle\wedge \mu_{\widetilde{I}}(x, a), \vee \gamma_{\widetilde{I}}(x, a)\right\rangle, \forall x \in X$, according to Definition 7. If $x \in X$, then $\widetilde{I}(x, a) \geq$ $\left\langle\wedge \mu_{\widetilde{I}}(x, a), \vee \gamma_{\widetilde{I}}(x, a)\right\rangle, \forall x \in X=\widetilde{A}(a)$. Thus, $x \in X^{* *}$; that is, $X \subseteq X^{* *}$.

On the other hand, assume that $\widetilde{B} \subseteq U^{B}$; then we can denote $\widetilde{B}=\left\{\left\langle\mu_{\widetilde{I}}(x, b), \gamma_{\widetilde{I}}(x, b)\right\rangle \mid b \in A\right\}$, where $\widetilde{B}(b)=$ $\langle 0,1\rangle$, if $b \in A-B$. And we can obtain that $\widetilde{B}^{*}=\{x \in$ $U \mid \widetilde{I}(x, b) \geq \widetilde{B}(b), \forall b \in A\}$, which follows that for any $x \in \widetilde{B}^{*}$ and $b \in B, \widetilde{I}(x, b) \geq \widetilde{B}(b)$ holds. So, we can obtain that $\widetilde{B}^{* *}(b)=\left\langle\wedge \mu_{\widetilde{I}}(x, b), \vee \gamma_{\widetilde{I}}(x, b)\right\rangle, \forall x \in$ $\widetilde{B}^{*} \geq \widetilde{B}(b)$. Hence, $\widetilde{B} \subseteq \widetilde{B}^{* *}$.

(3) It is obvious from (1) and (2).

(4) From (1) we can have $X \subseteq \widetilde{B}^{*} \Rightarrow \widetilde{B}^{* *} \subseteq X^{*}$, and from (2) we conclude $\widetilde{B} \subseteq \widetilde{B}^{* *} \Rightarrow \widetilde{B} \subseteq X^{*}$.
(5) It is obvious that $\left(\widetilde{B}_{1} \cup \widetilde{B}_{2}\right)^{*} \subseteq \widetilde{B}_{1}^{*} \cap \widetilde{B}_{2}^{*}$. Furthermore, $\forall x \in \widetilde{B}_{1}^{*} \cap \widetilde{B}_{2}^{*} \Leftrightarrow x \in \widetilde{B}_{1}^{*} \wedge x \in \widetilde{B}_{2}^{*} \Leftrightarrow \tilde{I}(x, b) \geq$ $\widetilde{B}(b), \forall b \in B_{1}$ and $\widetilde{I}(x, b) \geq \widetilde{B}(b), \forall b \in B_{2} \Leftrightarrow$ $\widetilde{I}(x, b) \geq \widetilde{B}(b), \forall b \in B_{1} \cup B_{2} \Leftrightarrow x \in\left(\widetilde{B}_{1} \cup \widetilde{B}_{2}\right)^{*} \Rightarrow$ $\widetilde{B}_{1}^{*} \cap \widetilde{B}_{2}^{*} \subseteq\left(\widetilde{B}_{1} \cup \widetilde{B}_{2}\right)^{*}$. Hence, $\left(\widetilde{B}_{1} \cup \widetilde{B}_{2}\right)^{*}=\widetilde{B}_{1}^{*} \cap \widetilde{B}_{2}^{*}$. $\left(X_{1} \cup X_{2}\right)^{*}=X_{1}^{*} \cap X_{2}^{*}$ can be obtained similarly.

(6) It can be easily proved from (1).

Definition 10. Let $(U, A, \widetilde{I})$ be an IF formal context. A pair $(X, \widetilde{B})$ is called an IF formal concept (in brief a concept) if $X^{*}=\widetilde{B}$ and $X=\widetilde{B}^{*}$ for $X \subseteq U, B \subseteq A$. $X$ and $\widetilde{B}$ are called the extension and the intension of $(X, \widetilde{B})$, respectively.

From the above, it is clear that both $\left(X^{* *}, X^{*}\right)$ and $\left(\widetilde{B}^{*}, \widetilde{B}^{* *}\right)$ are concepts.

IF concept lattice $L(U, A, \widetilde{I})$ is referred to as all concepts of an IF formal context $(U, A, \widetilde{I})$, and they are ordered by

$$
\left(X_{1}, \widetilde{B}_{1}\right) \leq\left(X_{2}, \widetilde{B}_{2}\right) \Longleftrightarrow X_{1} \subseteq X_{2} \Longleftrightarrow \widetilde{B}_{1} \supseteq \widetilde{B}_{2}
$$

where $\left(X_{1}, \widetilde{B}_{1}\right)$ and $\left(X_{2}, \widetilde{B}_{2}\right)$ are concepts. $\left(X_{1}, \widetilde{B}_{1}\right)$ is called a subconcept of $\left(X_{2}, \widetilde{B}_{2}\right)$, and $\left(X_{2}, \widetilde{B}_{2}\right)$ is called a superconcept of $\left(X_{1}, \widetilde{B}_{1}\right)$.

And we denote the family of all IF concept lattices by $\mathscr{L}=$ $\{L(U, A, \widetilde{I}) \mid(U, A, \widetilde{I})$ is an IF formal context $\}$.

Proposition 11. If $\left(X_{1}, \widetilde{B}_{1}\right)$ and $\left(X_{2}, \widetilde{B}_{2}\right)$ are two concepts of an IF formal context $(U, A, \widetilde{I})$, then both $\left(X_{1} \cap X_{2},\left(B_{1} \cup B_{2}\right)^{* *}\right)$ and $\left(\left(X_{1} \cup X_{2}\right)^{* *}, B_{1} \cap B_{2}\right)$ are also concepts.

Proof. It is straight from the definition and Proposition 9.

Hence, from the above, if the meet and join are given by

$$
\begin{aligned}
& \left(X_{1}, \widetilde{B}_{1}\right) \wedge\left(X_{2}, \widetilde{B}_{2}\right)=\left(X_{1} \cap X_{2},\left(\widetilde{B}_{1} \cup \widetilde{B}_{2}\right)^{* *}\right), \\
& \left(X_{1}, \widetilde{B}_{1}\right) \vee\left(X_{2}, \widetilde{B}_{2}\right)=\left(\left(X_{1} \cup X_{2}\right)^{* *}, B_{1} \cap \widetilde{B}_{2}\right),
\end{aligned}
$$

then the IF concept lattice $L(U, A, \widetilde{I})$ is complete lattice.

Example 12. In Example 8, we can find all concepts of the IF formal context by the definition, which are $\left(1, \widetilde{A}_{1}\right),\left(2, \widetilde{A}_{2}\right)$, $\left(4, \widetilde{A}_{3}\right),\left(12, \widetilde{A}_{4}\right),\left(13, \widetilde{A}_{5}\right),\left(24, \widetilde{A}_{6}\right),\left(123, \widetilde{A}_{7}\right),\left(124, \widetilde{A}_{8}\right)$, $\left(U, \widetilde{A}_{9}\right),(\emptyset, \widetilde{A})$, respectively, and we denote objects set $\left\{x_{i}, x_{j}\right\}$ by $i j(i, j=1,2,3,4)$ which is same to others, where

$$
\begin{gathered}
\widetilde{A}_{1}=\{(a, 0.9,0.0),(b, 0.7,0.2),(c, 0.2,0.5), \\
(d, 0.9,0.1),(e, 0.8,0.1)\}, \\
\widetilde{A}_{2}=\{(a, 0.8,0.1),(b, 0.8,0.2),(c, 0.8,0.1), \\
(d, 0.3,0.5),(e, 0.2,0.7)\}, \\
\widetilde{A}_{3}=\{(a, 0.7,0.2),(b, 0.8,0.1),(c, 0.7,0.1), \\
(d, 0.2,0.6),(e, 0.2,0.8)\},
\end{gathered}
$$




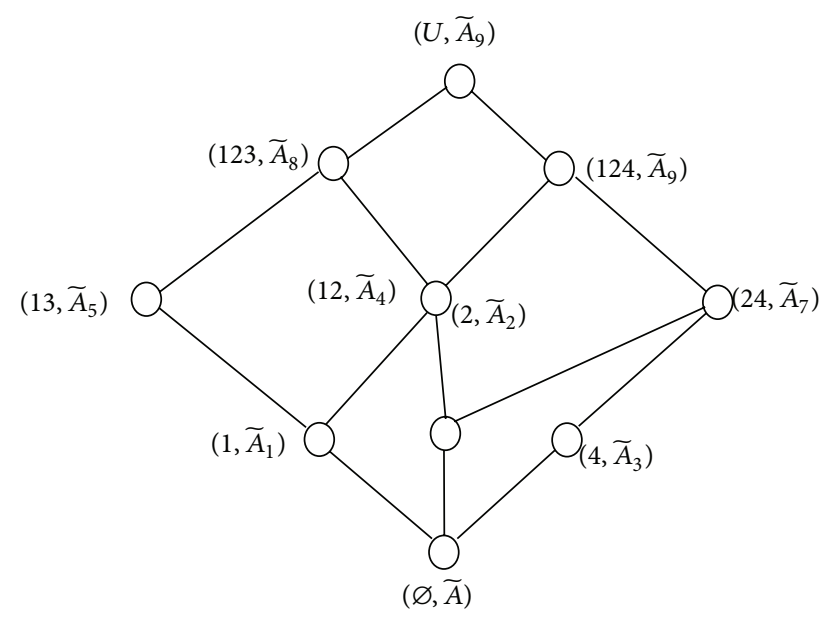

FIgURE 1: Concept lattice of the IF formal context $(U, A, \widetilde{I})$.

$$
\begin{aligned}
& \widetilde{A}_{4}=\{(a, 0.8,0.1),(b, 0.7,0.2),(c, 0.2,0.5),(d, 0.3,0.5),(e, 0.2,0.7)\}, \\
& \widetilde{A}_{5}=\{(a, 0.1,0.8),(b, 0.2,0.6),(c, 0.1,0.9), \\
&(d, 0.8,0.2),(e, 0.2,0.6)\}, \\
& \widetilde{A}_{6}=\{(a, 0.7,0.2),(b, 0.8,0.2),(c, 0.7,0.1), \\
&\quad(d, 0.2,0.6),(e, 0.2,0.8)\}, \\
& \widetilde{A}_{7}=\{(a, 0.1,0.8),(b, 0.2,0.6),(c, 0.1,0.9), \\
&(d, 0.3,0.5),(e, 0.2,0.7)\}, \\
& \widetilde{A}_{8}=\{(a, 0.7,0.2),(b, 0.7,0.2),(c, 0.2,0.5), \\
&\quad(d, 0.2,0.6),(e, 0.2,0.8)\}, \\
& \widetilde{A}_{9}=\{(a, 0.1,0.8),(b, 0.2,0.6),(c, 0.1,0.9), \\
&\quad(d, 0.2,0.6),(e, 0.2,0.8)\}, \\
& \widetilde{A}=\{(a, 1,0),(b, 1,0),(c, 1,0), \\
&(d, 1,0),(e, 1,0)\} .
\end{aligned}
$$

Furthermore, we can obtain the following IF concept lattice of the IF context (Figure 1).

\section{Attribute Reduction in IF Concept Lattices}

In this section, we discuss attribute reduction in IF concept lattices and obtain some propositions in a similar way to Zhang et al. [40] and Li and Zhang [28].

Definition 13. Let $L\left(U, A_{1}, \widetilde{I}_{1}\right)$ and $L\left(U, A_{2}, \widetilde{I}_{2}\right)$ be two IF concept lattices. If for any $(X, \widetilde{B}) \in L\left(U, A_{2}, \widetilde{I}_{2}\right)$, there exists $\left(X^{\prime}, \widetilde{B}^{\prime}\right) \in L\left(U, A_{1}, \widetilde{I}_{1}\right)$ such that $X=X^{\prime}$, then we say that $L\left(U, A_{2}, \widetilde{I}_{2}\right)$ is coarser than $L\left(U, A_{1}, \widetilde{I}_{1}\right)$ or $L\left(U, A_{1}, \widetilde{I}_{1}\right)$ is thinner than $L\left(U, A_{2}, \widetilde{I}_{2}\right)$, denoted by

$$
L\left(U, A_{1}, \widetilde{I}_{1}\right) \leq L\left(U, A_{2}, \widetilde{I}_{2}\right)
$$

If $L\left(U, A_{1}, \widetilde{I}_{1}\right) \leq L\left(U, A_{2}, \widetilde{I}_{2}\right)$ and $L\left(U, A_{2}, \widetilde{I}_{2}\right) \leq$ $L\left(U, A_{1}, \widetilde{I}_{1}\right)$, we say that $L\left(U, A_{1}, \widetilde{I}_{1}\right)$ and $L\left(U, A_{2}, \widetilde{I}_{2}\right)$ are isomorphic with each other and denoted by $L\left(U, A_{1}, \widetilde{I}_{1}\right) \cong$ $L\left(U, A_{2}, \widetilde{I}_{2}\right)$.

Definition 14. Let $(U, A, \widetilde{I})$ be an IF formal context. The set of all extensions of $L(U, A, \widetilde{I})$ is defined to be

$$
L_{U}(U, A, \widetilde{I})=\{X \mid(X, \widetilde{B}) \in L(U, A, \widetilde{I})\} .
$$

Let $(U, A, \widetilde{I})$ be an IF formal context and $D \subseteq A$. We denote $\widetilde{I}_{D}=\widetilde{I} \cap \widetilde{I}^{\prime}$, where $\widetilde{I}^{\prime}$ is an IF set of $U \times D$; that is, $\widetilde{I}_{D}=\left\{\left\langle(x, a), \mu_{\widetilde{I}_{D}}(x, a), \gamma_{\widetilde{I}_{D}}(x, a)\right\rangle \mid(x, a) \in U \times D\right\}$. Obviously, $\left(U, D, \widetilde{I}_{D}\right)$ is also an IF formal context, we denote all concepts of $\left(U, D, \widetilde{I}_{D}\right)$ by $L\left(U, D, \widetilde{I}_{D}\right)$ similarly. For any $(X, \widetilde{B}) \in L\left(U, D, \widetilde{I}_{D}\right)$, it satisfies that if $a \in D, X^{*} D(a)=X^{*}(a)$, otherwise $X^{* D}(a)=\langle 0,1\rangle$, and $\widetilde{B}^{*_{D}}=\{x \in U \mid \widetilde{I}(x, b) \geq$ $\widetilde{B}(b), \forall b \in D\}$.

Proposition 15. Let $(U, A, \widetilde{I})$ be an IF formal context. If $X \subseteq$ $U, D \subseteq A$ and $\widetilde{B} \in \mathscr{F} \mathscr{F}(U)$, then $X^{*_{D}} \subseteq X^{*}$ and $\widetilde{B}^{*} \subseteq \widetilde{B}^{{ }^{D}}$ hold.

Proof. They can be easily obtained from Proposition 9 and Definition 14.

Proposition 16. Let $(U, A, \widetilde{I})$ be an IF formal context. If $D \subseteq A$ and $D \neq \emptyset$, then there must exist the following relation:

$$
L(U, A, \widetilde{I}) \leq L\left(U, D, \widetilde{I}_{D}\right)
$$

Proof. For any $(X, \widetilde{B}) \in L\left(U, D, \widetilde{I}_{D}\right), X^{{ }^{*} D}=\widetilde{B}$ and $\widetilde{B}^{{ } D}=$ $X$. From the above discussions, we know that $\left(X^{* *}, X^{*}\right) \in$ $L(U, A, \widetilde{I})$ is concept; thus we only need to verify $X^{* *}=X$.

According to Proposition 9 (2), we can obtain that $X^{* *} \supseteq$ $X$. In addition to Proposition $15, \widetilde{B}=X^{*} D \subseteq X^{*} \Rightarrow X^{* *} \subseteq$ $\widetilde{B}^{*} \subseteq \widetilde{B}^{*}=X$. Therefore, $L(U, A, \widetilde{I}) \leq L\left(U, D, \widetilde{I}_{D}\right)$.

Corollary 17. Let $(U, A, \widetilde{I})$ be an IF formal context. If $D \subseteq A$, $D \neq \emptyset$, then $L_{U}\left(U, D, \widetilde{I}_{D}\right) \subseteq L_{U}(U, A, \widetilde{I})$.

Definition 18. Let $(U, A, \widetilde{I})$ be an IF formal context, $D \subseteq A$. We say that $D$ is a consistent set of $L(U, A, \widetilde{I})$, if $L\left(U, D, \widetilde{I}_{D}\right) \cong$ $L(U, A, \widetilde{I})$. If $D$ is a consistent set, and for any $d \in D$, there exists $L\left(U, D-d, \widetilde{I}_{D-d}\right) \not \equiv L(U, A, \widetilde{I})$, then $D$ is called an attribute reduction of $L(U, A, \widetilde{I})$. The intersection set of all reductions is called the core of $L(U, A, \widetilde{I})$.

Obviously, we can obtain the following propositions by the above definition.

Proposition 19. For any $L(U, A, \widetilde{I})$, there must exist a reduction of it. 
Proof. This proposition is immediately obtained in a similar way to the $L(U, A, I)$.

Proposition 20. Let $(U, A, \widetilde{I})$ be an IF formal context. If $D \subseteq$ $A$ and $D \neq \emptyset$, then

$D$ is a consistent set $\Longleftrightarrow L\left(U, D, \widetilde{I}_{D}\right) \leq L(U, A, \widetilde{I})$.

Proof. According to Proposition 15 and Definition 18, the conclusion can be obtained easily.

Definition 21. Let $(U, A, \widetilde{I})$ be an IF formal context and suppose that $\tau$ is an index set and all the reductions are denoted by $\left\{D^{i} \mid D^{i}\right.$ is a reduction, $\left.i \in \tau\right\}$. Then, attributes can be divided into four types as follows:

(1) absolutely necessary attribute (core attribute) $b: b \in$ $\cap_{i \in \tau} D^{i}$

(2) relatively necessary attribute $c: c \in \cup_{i \in \tau} D^{i}-\cap_{i \in \tau} D^{i}$;

(3) absolutely unnecessary attribute $d: d \in A-\cup_{i \in \tau} D^{i}$;

(4) unnecessary attribute $e: e \in A-\cap_{i \in \tau} D^{i}$.

In general, the reduction of $(U, A, \widetilde{I})$ is not unique. An example will be used to illustrate the above discussions as follows.

Example 22. For the IF formal context in Table 1, if we take out $\{a, c\}$ from the attributes set $A$, then we can obtain a new IF formal context $\left(U, D^{1}, \widetilde{I}_{D^{1}}\right)$, where $D^{1}=$ $A-\{a, c, d\}$. And we can get all concepts of $\left(U, D^{1}, \widetilde{I}_{D^{1}}\right)$, they are $\left(1, \widetilde{D}_{1}^{1}\right),\left(2, \widetilde{D}_{2}^{1}\right),\left(4, \widetilde{D}_{3}^{1}\right),\left(12, \widetilde{D}_{4}^{1}\right),\left(13, \widetilde{D}_{5}^{1}\right),\left(24, \widetilde{D}_{6}^{1}\right)$, $\left(123, \widetilde{D}_{7}^{1}\right),\left(124, \widetilde{D}_{8}^{1}\right),\left(U, \widetilde{D}_{9}^{1}\right)$, and $\left(\emptyset, \widetilde{D}^{1}\right)$ marked by $\mathrm{IFCl}^{\prime}$, IFC2 ${ }^{\prime}, \mathrm{FC}^{\prime}{ }^{\prime}, \mathrm{IFC}^{\prime}{ }^{\prime}, \mathrm{IFC}^{\prime}$, IFC6 ${ }^{\prime}, \mathrm{IFC}^{\prime}, \mathrm{IFC}^{\prime}$, IFC9 ${ }^{\prime}$, and IFC10', respectively, where

$$
\begin{gathered}
\widetilde{D}_{1}^{1}=\{(b, 0.7,0.2),(e, 0.8,0.1)\} \\
\widetilde{D}_{2}^{1}=\{(b, 0.8,0.2),(e, 0.2,0.7)\} \\
\widetilde{D}_{3}^{1}=\{(b, 0.8,0.1),(e, 0.2,0.8)\} \\
\widetilde{D}_{4}^{1}=\{(b, 0.7,0.2),(e, 0.2,0.7)\} \\
\widetilde{D}_{5}^{1}=\{(b, 0.2,0.6),(e, 0.2,0.6)\} \\
\widetilde{D}_{6}^{1}=\{(b, 0.7,0.2),(e, 0.2,0.8)\} \\
\widetilde{D}_{7}^{1}=\{(b, 0.8,0.2),(e, 0.2,0.8)\} \\
\widetilde{D}_{8}^{1}=\{(b, 0.2,0.6),(e, 0.2,0.7)\}, \\
\widetilde{D}_{9}^{1}=\{(b, 0.7,0.2),(e, 0.2,0.8)\}, \\
\widetilde{D}^{1}=\{(b, 1,0),(e, 1,0)\} .
\end{gathered}
$$

In addition, we can obtain concept lattice of context $L\left(U, D^{1}, \widetilde{I}_{D}^{1}\right)$, as shown in Figure 2.

From Figures 1 and 2 , we can find easily that $L\left(U, D^{1}, \widetilde{I}_{D^{1}}\right)$ and $L(U, A, \widetilde{I})$ are isomorphic. So, $D^{1}$ is a consistent set of

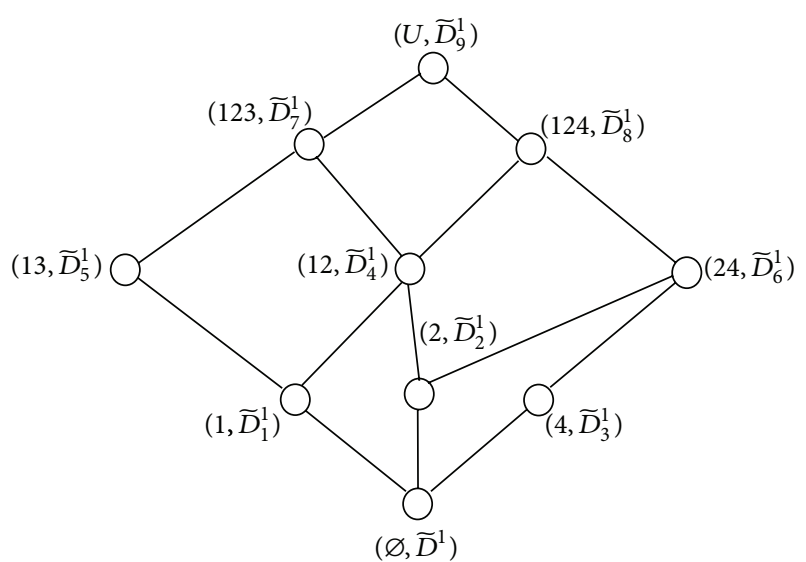

FIgURE 2: Concept lattice of the IF formal context $(U, A, \widetilde{I})$.

$(U, A, \widetilde{I})$. In fact, we can find that $L\left(U, D^{1}-b, \widetilde{I}_{D^{1}-b}\right) \not$ $L(U, A, \widetilde{I}), L\left(U, D^{1}-e, \widetilde{I}_{D^{1}-e}\right) \not L(U, A, \widetilde{I})$ by calculation. Hence, $D^{1}$ is a reduction of $(U, A, \widetilde{I})$.

If we take out $\{a, c, e\}$ from the attributes set $A$, then we can obtain a new IF formal context $\left(U, D^{2}, \widetilde{I}_{D^{2}}\right)$, where $D^{2}=A-\{a, c, e\}$. And we can get all concepts of $\left(U, D^{2}, \widetilde{I}_{D^{2}}\right)$, which are $\left(1, \widetilde{D}_{1}^{2}\right),\left(2, \widetilde{D}_{2}^{2}\right),\left(4, \widetilde{D}_{3}^{2}\right),\left(12, \widetilde{D}_{4}^{2}\right),\left(13, \widetilde{D}_{5}^{2}\right),\left(24, \widetilde{D}_{6}^{2}\right)$, $\left(123, \widetilde{D}_{7}^{2}\right),\left(124, \widetilde{D}_{8}^{2}\right),\left(U, \widetilde{D}_{9}^{2}\right)$, and $\left(\emptyset, \widetilde{D}^{2}\right)$, respectively, where

$$
\begin{gathered}
\widetilde{D}_{1}^{2}=\{(b, 0.7,0.2),(d, 0.9,0.1)\}, \\
\widetilde{D}_{2}^{2}=\{(b, 0.8,0.2),(d, 0.3,0.5)\}, \\
\widetilde{D}_{3}^{2}=\{(b, 0.8,0.1),(d, 0.2,0.6)\}, \\
\widetilde{D}_{4}^{2}=\{(b, 0.7,0.2),(d, 0.3,0.5)\}, \\
\widetilde{D}_{5}^{2}=\{(b, 0.2,0.6),(d, 0.8,0.2)\}, \\
\widetilde{D}_{6}^{2}=\{(b, 0.8,0.2),(d, 0.2,0.6)\}, \\
\widetilde{D}_{7}^{2}=\{(b, 0.2,0.6),(d, 0.3,0.5)\}, \\
\widetilde{D}_{8}^{2}=\{(b, 0.7,0.2),(d, 0.2,0.6)\}, \\
\widetilde{D_{9}^{2}}=\{(b, 0.2,0.6),(d, 0.2,0.6)\}, \\
\widetilde{D}^{2}=\{(b, 1,0),(d, 1,0)\} .
\end{gathered}
$$

Obviously, $L\left(U, D^{2}, \widetilde{I}_{D^{2}}\right)$ is isomorphic with $L(U, A, \widetilde{I})$.

Corollary 23. The core is the reduction $\Leftrightarrow$ The reduction is only one.

Proof. $\Leftarrow$ Obviously.

$\Rightarrow$ Assume that the core is the reduction, and the reduction is not unique; that is, there are two reductions: $D^{i} \neq D^{j}$ at least. Hence, the core of the reductions $\cap D^{t} \subseteq D^{i} \cap D^{j} \subset D^{i}$. For $D^{i}$ is the reduction, the proper subset of it (where it is the core of the reductions) must not be the reduction. This clearly contradicts the known conditions. So, if the core is the reduction, the reduction is only one. 
Obviously, the following corollaries can be obtained by the above definitions and propositions.

Corollary 24. Let $(U, A, \widetilde{I})$ be an IF formal context; $a \in A$ is a core attribute $\Leftrightarrow A-\{a\}$ is not a consistent set.

Corollary 25. Let $(U, A, \widetilde{I})$ be an IF formal context, $a \in A$ is an unnecessary attribute $\Leftrightarrow A-\{a\}$ is a consistent set.

Since the reduction $D$ of an IF formal context satisfies the following conditions: (1) $D \subseteq A$ a consistent set; (2) $\forall d \in D, D \backslash$ $\{d\}$ is not a consistent set; it is helpful to give the necessary and sufficient conditions of consistent sets in order to get reductions more easily.

Proposition 26. Let $(U, A, \widetilde{I})$ be an IF formal context, $D \subseteq$ $A, D \neq \emptyset$.

Then $D$ is a consistent set of $(U, A, \widetilde{I})$

$$
\Longleftrightarrow \widetilde{B}^{* *_{D}{ }^{*} D}=\widetilde{B}^{*} \text { for any } \widetilde{B} \in U^{A} \text {. }
$$

Proof. Assume that $D$ is a consistent set; then we have $L\left(U, D, \widetilde{I}_{D}\right) \leq L(U, A, \widetilde{I})$ according to Proposition 20 . For any $\widetilde{B} \in U^{A}$, it is easy to see that $\left(\widetilde{B}^{*}, \widetilde{B}^{* *}\right) \in L(U, A, \widetilde{I})$. Thus, by Definition 13 , there exists $\widetilde{B}^{\prime} \in U^{D}$ such that $\left(\widetilde{B}^{*}, \widetilde{B}^{\prime}\right) \in$ $L\left(U, D, \widetilde{I}_{D}\right)$. Hence, $\widetilde{B}^{\prime}=\widetilde{B}^{* *_{D}}$ and $\widetilde{B}^{*}=\widetilde{B}^{\prime *_{D}}$, which concludes $\widetilde{B}^{* * D^{*} D}=\widetilde{B}^{*}$.

Conversely, suppose that $\widetilde{B}^{*{ }^{*}{ }^{*}{ }_{D}}=\widetilde{B}^{*}$ for any $\widetilde{B} \in U^{A}$. To prove that $D$ is a consistent set of $(U, A, \widetilde{I})$, then it suffices to show that for any $(X, \widetilde{B}) \in L(U, A, \widetilde{I})$, there exists $\widetilde{B}^{\prime} \in U^{D}$ such that $\left(X, \widetilde{B}^{\prime}\right) \in L\left(U, D, \widetilde{I}_{D}\right)$. Thus, suppose that $(X, \widetilde{B}) \in$ $L(U, A, \widetilde{I})$; then we can get that $X^{*}=\widetilde{B}$ and $X=\widetilde{B}^{*}$. Taking $\widetilde{B}^{\prime}=\widetilde{B}^{* *}$, then we can obtain that $\widetilde{B}^{\prime{ }^{*} D}=\widetilde{B}^{* *} D^{*} D=\widetilde{B}^{*}=X$, and so $\left(X, \widetilde{B}^{\prime}\right) \in L\left(U, D, \widetilde{I}_{D}\right)$.

Corollary 27. Let $(U, A, \widetilde{I})$ be an IF formal context, $D \subseteq$ $A$, and $D \neq \emptyset$. Then $D$ is a consistent set of $(U, A, \widetilde{I}) \Leftrightarrow \forall \widetilde{B} \in$ $U^{A}, \exists \widetilde{B}^{\prime} \in U^{D}$ such that $\widetilde{B}^{\prime{ }^{*} D}=\widetilde{B}^{*}$.

Proof. It can be certified easily by Proposition 20 .

Proposition 28. Let $(U, A, \widetilde{I})$ be an IF formal context, $D \subseteq$ $A, D \neq \emptyset$, and $E=A-D$. Then, $D$ is a consistent set of $(U, A, \widetilde{I})$ if and only if $\forall \widetilde{B} \in U^{E}, \exists \widetilde{B}^{\prime} \in U^{D}$ such that $\widetilde{B}^{\prime{ }^{*} D}=\widetilde{B}^{*_{E}}$.

Proof. Suppose that $D$ is a consistent set; then $\widetilde{B}_{1}^{*}=\widetilde{B}_{1}^{*} D$ for any $\widetilde{B}_{1} \in U^{A}$. For any $\widetilde{B} \in U^{E}$, let $\widetilde{B}_{2}=\widetilde{B} \cup \widetilde{B}_{1}$, with $\widetilde{B}_{1} \in U^{D}$, $\widetilde{B}_{1}(a)=\left\langle\wedge_{\forall x \in U} \mu_{\widetilde{I}}(x, a), \vee_{\forall x \in U} \gamma_{\widetilde{I}}(x, a)\right\rangle$, for $a \in D$; then $\widetilde{B}_{1}^{*}=$ $\widetilde{B}^{{ }_{E} E}$. Thus, there exists $\widetilde{B}^{\prime} \in U^{D}$ such that then $\widetilde{B}^{\prime *_{D}}=\widetilde{B}^{{ }^{*} E}$ by Corollary 25.

Suppose that there exists $\widetilde{B}^{\prime} \in U^{D}$ such that $\widetilde{B}^{*_{D}}=\widetilde{B}^{*_{E}}$ for any $\widetilde{B} \in U^{E}$. Let $\left(X, \widetilde{B}_{2}\right) \in L(U, A, \widetilde{I})$; then $X^{*}=\widetilde{B}_{2}$ and $X=$ $\widetilde{B}_{2}^{*}$. Let $\widetilde{B}_{2}=\widetilde{B} \cup \widetilde{B}_{1}$ with $\widetilde{B} \in U^{E}$ and $\widetilde{B}_{1} \in U^{D}$, then $\widetilde{B}_{2}^{*}=(\widetilde{B} \cup$ $\left.\widetilde{B}_{1}\right)^{*}=\widetilde{B}^{*} \cap \widetilde{B}_{1}^{*}=\widetilde{B}^{{ }^{D} D} \cap \widetilde{B}_{1}^{* E}$. Hence, there exists $\widetilde{B}^{\prime} \in U^{D}$ such that $\widetilde{B}^{\prime *_{D}}=\widetilde{B}_{1}^{*_{E}}$, so $\widetilde{B}^{*_{D}} \cap \widetilde{B}_{1}^{*_{E}}=\widetilde{B}_{1}^{{ }^{*} D} \cap \widetilde{B}^{\prime{ }^{*} D}=\left(\widetilde{B}_{1} \cup \widetilde{B}^{\prime}\right)^{*_{D}}=X$. Now it follows from Proposition 9 (3) and Definition 10 that
$\left(\left(\widetilde{B}_{1} \cup \widetilde{B}^{\prime}\right)^{*} D,\left(\widetilde{B}_{1} \cup \widetilde{B}^{\prime}\right)^{*^{*}{ }^{*} D}\right) \in L\left(U, D, \widetilde{I}_{D}\right)$, and so $L\left(U, D, \widetilde{I}_{D}\right) \leq$ $L(U, A, \widetilde{I})$, which means that $D$ is a consistent set.

The functions of attributes, which are closely related to consistent sets, vary from one to another. So, sufficient conditions to determine the type of attributes are useful in attribute reduction.

Proposition 29. Let $(U, A, \widetilde{I})$ be an IF formal context. Then, a is an absolutely necessary attribute if there exists $x_{i}, x_{j} \in U$ such that $\widetilde{I}\left(x_{i}, a\right)>\widetilde{I}\left(x_{j}, a\right)$, and for any $b \neq a \widetilde{I}\left(x_{i}, b\right) \leq$ $\widetilde{I}\left(x_{j}, b\right)$.

Proof. Suppose that $a$ is an unnecessary attribute, then $D=$ $A-\{a\}$ is a consistent set; that is, $L\left(U, D, \widetilde{I}_{D}\right) \cong L(U, A, \widetilde{I})$. Let $\widetilde{B}=x_{i}^{*}$, then $\left(\widetilde{B}^{*}, \widetilde{B}^{* *}\right) \in L(U, A, \widetilde{I})$. Since $\widetilde{I}\left(x_{i}, a\right)>$ $\widetilde{I}\left(x_{j}, a\right)$, we know that $x_{i} \in \widetilde{B}^{*}$ and $x_{j} \notin \widetilde{B}^{*}$. Assume that $\left(X, \widetilde{B}^{\prime}\right) \in L\left(U, D, \widetilde{I}_{D}\right)$ and $x_{i} \in X$, then $X^{*_{D}}=\widetilde{B}^{\prime}, \widetilde{B}^{\prime *_{D}}=X$. From $x_{i} \in X$, we know that $\widetilde{B}^{\prime}=X^{*} D \subseteq x_{i}^{* D}$. But since for any $b \neq a, \widetilde{I}\left(x_{i}, b\right) \leq \widetilde{I}\left(x_{j}, b\right)$. It follows that $\widetilde{B}^{\prime}(b) \leq$ $\widetilde{I}\left(x_{j}, b\right)$ and so $x_{j} \in X$. That is to say, in IF formal context $\left(U, D, \widetilde{I}_{D}\right)$, every concept which contains $x_{i}$ also contains $x_{j}$. But $\widetilde{I}\left(x_{i}, a\right)>\widetilde{I}\left(x_{j}, a\right)$. Consistently, there is no concept in $L\left(U, D, \widetilde{I}_{D}\right)$ whose extent is equal to $\widetilde{B}^{*}$. Thus, $D$ is not a consistent set, which gets a contradiction. Therefore, $a$ is an absolutely necessary attribute.

Proposition 30. Let $(U, A, \widetilde{I})$ be an IF formal context. Then, $a$ is an unnecessary attribute if the following conditions hold: for any $x_{i}, x_{j} \in U$, if $\widetilde{I}\left(x_{i}, a\right)>\widetilde{I}\left(x_{j}, a\right)$, then there exists $b \neq a$ such that $\widetilde{I}\left(x_{i}, b\right)>\widetilde{I}\left(x_{j}, b\right)$. Moreover, if there exists $x_{k} \in A$ such that $\widetilde{I}\left(x_{k}, a\right) \geq \widetilde{I}\left(x_{i}, a\right)$, then $\widetilde{I}\left(x_{k}, b\right) \geq \widetilde{I}\left(x_{j}, b\right)$.

Proof. Suppose that $a=a_{l}, D=A-\left\{a_{l}\right\}$. It suffices to prove that $D$ is consistent set. By Corollary 27 , it remains to prove that for any $\widetilde{B} \in U^{A}$, there exists $\widetilde{B}^{\prime} \in U^{D}$ such that $\widetilde{B}^{\prime *_{D}}=$ $\widetilde{B}^{*}$. So suppose that $\widetilde{B}=\left\{\widetilde{I}\left(x_{s 1}, a_{1}\right), \widetilde{I}\left(x_{s 2}, a_{2}\right), \ldots, \widetilde{I}\left(x_{s m}, a_{m}\right)\right\}$, where $x_{s t} \in U a_{t} \in A, 1 \leq s \leq|U|, 1 \leq t \leq m$.

If for any $x \in U, \widetilde{I}\left(x, a_{l}\right) \geq \widetilde{I}\left(x_{s l}, a_{l}\right)$, then let $\widetilde{B}^{\prime}=\left\{\widetilde{I}\left(x_{s 1}\right.\right.$, $\left.\left.a_{1}\right), \widetilde{I}\left(x_{s 2}, a_{2}\right), \ldots, \widetilde{I}\left(x_{s l-1}, a_{l-1}\right), \widetilde{I}\left(x_{s l+1}, a_{l+1}\right), \ldots, \widetilde{I}\left(x_{s m}, a_{m}\right)\right\}$, and so we can get that $\widetilde{B}^{\prime *_{D}}=\widetilde{B}^{*}$

Otherwise, assume that there are $\left\{x_{t 1}, x_{t 2}, \ldots, x_{t \alpha}\right\} \subseteq U$, such that $\widetilde{I}\left(x_{s l}, a_{l}\right)>\widetilde{I}\left(x_{t \beta}, a_{l}\right)(1 \leq \beta \leq \alpha)$; then there exist, according the condition, $a_{q 1}, a_{q 2}, \ldots, a_{q \eta} \in A-\left\{a_{l}\right\}$ such that $\widetilde{I}\left(x_{s l}, a_{q \sigma}\right)>\widetilde{I}\left(x_{t \beta}, a_{q \sigma}\right)(1 \leq \sigma \leq \eta)$. Moreover, if there exists $x_{k}$ such that $\widetilde{I}\left(x_{k}, a_{l}\right) \geq \widetilde{I}\left(x_{s l}, a_{l}\right)$, then $\widetilde{I}\left(x_{k}, a_{q \sigma}\right) \geq \widetilde{I}\left(x_{s l}, a_{q \sigma}\right)$. Let $\widetilde{B}^{\prime}=\widetilde{B}_{1} \cup \widetilde{B}_{2}$, where

$$
\begin{aligned}
\widetilde{B}_{1}=\left\{\widetilde{I}\left(x_{s 1}, a_{1}\right), \widetilde{I}\left(x_{s 2}, a_{2}\right), \ldots, \widetilde{I}\left(x_{s l-1}, a_{l-1}\right),\right. \\
\left.\widetilde{I}\left(x_{s l+1}, a_{l+1}\right), \ldots, \widetilde{I}\left(x_{s m}, a_{m}\right)\right\}, \\
\widetilde{B}_{2}=\left\{\widetilde{I}\left(x_{s l}, a_{q 1}\right), \widetilde{I}\left(x_{s l}, a_{q 2}\right), \ldots, \widetilde{I}\left(x_{s l}, a_{q \eta}\right)\right\} ;
\end{aligned}
$$


then it follows that $\widetilde{B}^{\prime{ }^{*} D}=\widetilde{B}_{1}^{*} \cap \cap \widetilde{B}_{2}^{*}$. So we know that if $x \in \widetilde{B}^{*}$, that is, $\widetilde{I}\left(x, a_{t}\right) \geq \widetilde{I}\left(x_{s t}, a_{t}\right)$, then $\widetilde{I}\left(x, a_{q \sigma}\right) \geq \widetilde{I}\left(x_{s l}, a_{q \sigma}\right)$. Then, $x \in \widetilde{B}_{1}^{*}$ and $x \in \widetilde{B}_{2}^{*}$. It follows that $x \in \widetilde{B}^{\prime{ }^{*} D}$ and so $\widetilde{B}^{*} \subseteq \widetilde{B}^{\prime *} D$. If $x \notin \widetilde{B}^{*}$, that is, there exists $a_{t_{0}} \in A$ such that $\widetilde{I}\left(x_{s t}, a_{t_{0}}\right)>\widetilde{I}\left(x, a_{t_{0}}\right)$. If $a_{t_{0}}=a_{l}$, that is, $x \in\left\{x_{t 1}, x_{t 2}, \ldots, x_{t \alpha}\right\}$, then $\widetilde{I}\left(x_{s l}, a_{q \sigma}\right)>\widetilde{I}\left(x, a_{q \sigma}\right)$; that is, $x \notin \widetilde{B}_{2}^{* D}$, and so $x \notin \widetilde{B}^{\prime *_{D}}$. If $a_{t_{0}} \neq a_{l}$, that is, then $\widetilde{I}\left(x_{s t_{0}}, a_{t_{0}}\right)>\widetilde{I}\left(x, a_{t_{0}}\right)$; that is, $x \notin \widetilde{B}_{1}^{*_{D}}$, and so $x \notin \widetilde{B}^{\prime{ }^{*} D}$. Hence, we conclude that for any $\widetilde{B} \in U^{A}$ there exists $\widetilde{B}^{\prime} \in U^{D}$ such that $\widetilde{B}^{\prime *_{D}}=\widetilde{B}^{*}$.

Proposition 31. Let $(U, A, \widetilde{I})$ be an IF formal context. Then a is an unnecessary attribute if there exists $b \in A$ such that for any $x_{i}, x_{j} \in U, \widetilde{I}\left(x_{i}, a\right)>\widetilde{I}\left(x_{j}, a\right)$ implies that $\widetilde{I}\left(x_{i}, b\right)>\widetilde{I}\left(x_{j}, b\right)$. Moreover, if $b$ is an absolutely necessary attribute, then $a$ is an absolutely unnecessary attribute.

Proof. We denote $a=a_{1}, b=a_{l-1}$, and $D=A-\left\{a_{l}\right\}$. It suffices to prove that $D$ is consistent set. By Corollary 27, it remains to prove that for any $\widetilde{B} \in U^{A}$, there exists $\widetilde{B}^{\prime} \in U^{D}$, such that $\widetilde{B}^{\prime{ }^{*} D}=\widetilde{B}^{*}$. So suppose that $\widetilde{B}=$ $\left\{\widetilde{I}\left(x_{s 1}, a_{1}\right), \widetilde{I}\left(x_{s 2}, a_{2}\right), \ldots, \widetilde{I}\left(x_{s m}, a_{m}\right)\right\}$, where $x_{s t} \in U, a_{t} \in A$, $1 \leq s \leq|U|, 1 \leq t \leq m$.

If for any $x \in U, \widetilde{I}\left(x, a_{l}\right) \geq \widetilde{I}\left(x_{s l}, a_{l}\right)$, then let $\widetilde{B}^{\prime}=\left\{\widetilde{I}\left(x_{s 1}\right.\right.$, $\left.\left.a_{1}\right), \widetilde{I}\left(x_{s 2}, a_{2}\right), \ldots, \widetilde{I}\left(x_{s l-1}, a_{l-1}\right), \widetilde{I}\left(x_{s l+1}, a_{l+1}\right), \ldots, \widetilde{I}\left(x_{s m}, a_{m}\right)\right\}$, and it follows that $\widetilde{B}^{\prime *_{D}}=\widetilde{B}^{*}$.

Otherwise, there exists $x_{k}$ such that $\widetilde{I}\left(x_{s l}, a_{l}\right)>\widetilde{I}\left(x_{k}, a_{l}\right)$; then $\widetilde{I}\left(x_{s l}, a_{l-1}\right)>\widetilde{I}\left(x_{k}, a_{l-1}\right)$. Denote $\left\{x_{t 1}, x_{t 2}, \ldots, x_{t \alpha}\right\} \subseteq$ $U$ to be the set whose elements satisfy the condition that $\widetilde{I}\left(x_{t \beta}, a_{l}\right) \geq \widetilde{I}\left(x_{s l}, a_{l}\right)(1 \leq \beta \leq \alpha)$. Then, $\widetilde{I}\left(x_{t \beta}, a_{l}\right)>\widetilde{I}\left(x_{k}, a_{l}\right)$ and thus $\widetilde{I}\left(x_{t \beta}, a_{l-1}\right)>\widetilde{I}\left(x_{k}, a_{l-1}\right)$. Let $\widetilde{B}^{\prime}=\widetilde{B}_{1} \cup \widetilde{B}_{2}$, where

$$
\begin{aligned}
& \widetilde{B}_{1}=\left\{\widetilde{I}\left(x_{s 1}, a_{1}\right), \widetilde{I}\left(x_{s 2}, a_{2}\right), \ldots, \widetilde{I}\left(x_{s l-1}, a_{l-1}\right),\right. \\
&\left.\widetilde{I}\left(x_{s l+1}, a_{l+1}\right), \ldots, \widetilde{I}\left(x_{s m}, a_{m}\right)\right\}, \\
& \widetilde{B}_{2}=\left\{\widetilde{I}\left(x_{s 1}, a_{1}\right), \widetilde{I}\left(x_{s 2}, a_{2}\right), \ldots, \widetilde{I}\left(x_{s l-2}, a_{l-2}\right),\right. \\
& \\
&\left.\underset{1 \leq \beta \leq \alpha}{\wedge} \widetilde{I}\left(x_{t \beta}, a_{l-1}\right), \widetilde{I}\left(x_{s l+1}, a_{l+1}\right), \ldots, \widetilde{I}\left(x_{s m}, a_{m}\right)\right\},
\end{aligned}
$$

then it follows that $\widetilde{B}^{\prime *_{D}}=\widetilde{B}_{1}^{*} \cap \cap \widetilde{B}_{2}^{* D}$. So we know that if $x \in \widetilde{B}^{*}$, that is, for all $a_{t} \in A, \widetilde{I}\left(x, a_{t}\right) \geq \widetilde{I}\left(x_{s t}, a_{t}\right)$, then $x \in$ $B_{1}^{* D}$ and $x \in\left\{x_{t 1}, x_{t 2}, \ldots, x_{t \alpha}\right\}$. Then, $x \in \widetilde{B}_{1}^{*}$ and $x \in \widetilde{B}_{2}^{*}$. It follows that $x \in \widetilde{B}^{\prime *_{D}}$ and so $\widetilde{B}^{*} \subseteq \widetilde{B}^{\prime{ }^{*} D}$. If $x \notin \widetilde{B}^{*}$, then $x \notin B_{1}^{*_{D}}$ or $\widetilde{I}\left(x_{s l}, a_{l}\right)>\widetilde{I}\left(x, a_{l}\right)$. If $x \notin B_{1}^{*_{D}}$, then $x \in \widetilde{B}^{\prime *_{D}}$. If $\widetilde{I}\left(x_{s l}, a_{l}\right)>\widetilde{I}\left(x, a_{l}\right)$, then $\widetilde{I}\left(x_{t \beta}, a_{l-1}\right)>\widetilde{I}\left(x, a_{l-1}\right)$, and so, there exists $a_{t_{0}} \in A$ such that $\widetilde{I}\left(x_{s t}, a_{t_{0}}\right)>\widetilde{I}\left(x, a_{t_{0}}\right)$. If $a_{t_{0}}=a_{l}$, that is, $x \in\left\{x_{t 1}, x_{t 2}, \ldots, x_{t \alpha}\right\}$, then $\widetilde{I}\left(x_{s l}, a_{q \sigma}\right)>\widetilde{I}\left(x, a_{q \sigma}\right)$; that is, $x \notin \widetilde{B}_{2}^{*} D$, and so $\wedge_{1 \leq \beta \leq \alpha} \widetilde{I}\left(x_{t \beta}, a_{l-1}\right)>\widetilde{I}\left(x, a_{l-1}\right)$ that is, $x \notin B_{2}^{*} D$ and so $x \notin \widetilde{B}^{\prime{ }^{*} D}$. Thus, we conclude that for any $\widetilde{B} \in U^{A}$ there exists $\widetilde{B}^{\prime} \in U^{D}$ such that $\widetilde{B}^{\prime *_{D}}=\widetilde{B}^{*}$. Therefore $a_{l}$ is an unnecessary attribute.
Moreover, suppose that $a_{l-1}$ is an absolutely necessary attribute and $D$ is a consistent set which contains $a_{l-1}$. Since $a_{l-1}$ is an absolutely necessary attribute, we have $a_{l-1} \in$ $D$; thus $D-\left\{a_{l}\right\}$ is also a consistent set, that is, $D$ is not a reduction. Therefore, $a_{l}$ is an absolutely unnecessary attribute.

Corollary 32. Let $(U, A, \widetilde{I})$ be an IF formal context. Then, $a \in$ $A$ is an absolutely unnecessary attribute if for any $x_{i}, x_{j} \in U$, $\widetilde{I}\left(x_{j}, a\right) \geq \widetilde{I}\left(x_{i}, a\right)$.

\section{Approach to Reduction}

In this section, discernibility matrix and discernibility function $[18,41]$ are introduced to compute all reductions for an IF formal context based on the conclusions discussed in Section 4, and we discuss the approach to reduction as well as the corresponding characteristics. Furthermore, we also show corresponding reduction algorithm.

Definition 33. Let $(U, A, \widetilde{I})$ be an IF formal context and $X_{i}, X_{j} \in L_{U}(U, A, \widetilde{I}) \cup\left\{\left\{x_{j}\right\} \mid x_{j} \in U\right\}$, we define

$$
\begin{aligned}
& \mathscr{D}^{*}\left(X_{i}, X_{j}\right) \\
&= \begin{cases}\left\{a \in A \mid \mu_{X_{i}^{*}}(a)>\mu_{X_{j}^{*}}(a)\right. & \\
\text { or } \left.\gamma_{X_{i}^{*}}(a)<\gamma_{X_{j}^{*}}(a)\right\} & X_{i} \in L_{U}(U, A, \widetilde{I}), \\
\emptyset & X_{j}=\left\{x_{j}\right\} \not \subset X_{i} \\
\text { otherwise, }\end{cases} \\
& \mathscr{D}^{*}=\left\{\mathscr{D}^{*}\left(X_{i}, X_{j}\right) \mid X_{i}, X_{j} \in L_{U}(U, A, \widetilde{I}) \cup\left\{\left\{x_{j}\right\} \mid x_{j} \in U\right\}\right\} \\
&=\left(\mathscr{D}^{*}\left(X_{i}, X_{j}\right)\right)_{\left|L_{U}(U, A, \tilde{I}) \cup\left\{\left\{x_{j}\right\} \mid x_{j} \in U\right\}\right|^{2}}
\end{aligned}
$$

Then, $\mathscr{D}^{*}\left(X_{i}, X_{j}\right)$ is called discernibility attributes set between $X_{i}$ and $X_{j}$. And $\mathscr{D}^{*}$ is referred to as discernibility matrix of the IF formal context $(U, A, \widetilde{I})$.

Proposition 34. Let $(U, A, \widetilde{I})$ be an IF formal context and $D \subseteq$ $A, D \neq \emptyset$. Then, the following two propositions are equivalent.

(1) $D$ is a consistent set of $(U, A, \widetilde{I})$.

(2) If $\mathscr{D}^{*}\left(X_{i}, X_{j}\right) \neq \emptyset$, then $D \cap \mathscr{D}^{*}\left(X_{i}, X_{j}\right) \neq \emptyset$, for all $\mathscr{D}^{*}\left(X_{i}, X_{j}\right) \in \mathscr{D}^{*}$.

Proof. (1) $\Rightarrow$ (2) We assume that property (2) does not hold. That is, $\exists \mathscr{D}^{*}\left(X_{i}, X_{j}\right) \neq \emptyset \in \mathscr{D}^{*}, X_{j}=\left\{x_{j}\right\} \not \subset X_{i}$ such that $D \cap \mathscr{D}^{*}\left(X_{i}, X_{j}\right)=\emptyset$. That is to say for all $a \in D$ such that $\mu_{X_{i}^{*}}(a) \leq \mu_{X_{j}^{*}}(a)$ and $\gamma_{X_{i}^{*}}(a) \geq \gamma_{X_{j}^{*}}(a)$; hence $x_{j} \in X_{i}^{*^{D} *^{*} D}$. In other words, $\left(X_{i}, X_{i}^{*} D\right) \notin L\left(U, D, \widetilde{I}_{D}\right)$. It is paradoxical that $D$ is a consistent set of $(U, A, \widetilde{I})$.

(2) $\Rightarrow$ (1) If for all $\mathscr{D}^{*}\left(X_{i}, X_{j}\right) \neq \emptyset \in \mathscr{D}^{*}$, then for all $a \in$ $D \cap \mathscr{D}^{*}\left(X_{i}, X_{j}\right) \neq \emptyset$ such that $\mu_{X_{i}^{*}}(a)>\mu_{X_{j}^{*}}(a)$ or $\gamma_{X_{i}^{*} T}(a)<$ $\gamma_{X_{j}^{*} T}(a)$ and $\Rightarrow \mu_{X_{i}^{*} D}(a)>\mu_{X_{j}^{*} D}(a)$ or $\gamma_{X_{i}^{*} D}(a)<\gamma_{X_{j}^{*} D}(a)$. 
TABLE 2: Discernibility matrix in Example 22.

\begin{tabular}{lccccccccccccccc}
\hline$X_{i} / X_{j}$ & $x_{1}$ & $x_{2}$ & $x_{3}$ & $x_{4}$ & 1 & 2 & 4 & 12 & 13 & 24 & 123 & 124 & $U$ & $\emptyset$ \\
\hline$x_{1}$ & $\emptyset$ & $a d e$ & $A$ & $a d e$ & $\emptyset$ & $a d e$ & $a d e$ & $\emptyset$ & $\emptyset$ & $\emptyset$ & $\emptyset$ & $\emptyset$ & $\emptyset$ & $\emptyset$ \\
$x_{2}$ & $b c$ & $\emptyset$ & $a b c$ & $a c d e$ & $b c$ & $\emptyset$ & $a c d e$ & $\emptyset$ & $\emptyset$ & $\emptyset$ & $\emptyset$ & $\emptyset$ & $\emptyset$ & $\emptyset$ \\
$x_{3}$ & $\emptyset$ & $\emptyset$ & $\emptyset$ & $\emptyset$ & $\emptyset$ & $\emptyset$ & $\emptyset$ & $\emptyset$ & $\emptyset$ & $\emptyset$ & $\emptyset$ & $\emptyset$ & $\emptyset$ & $\emptyset$ \\
$x_{4}$ & $b c$ & $b$ & $a b c$ & $\emptyset$ & $b c$ & $b$ & $\emptyset$ & $\emptyset$ & $\emptyset$ & $\emptyset$ & $\emptyset$ & $\emptyset$ & $\emptyset$ & $\emptyset$ \\
1 & $\emptyset$ & $a d e$ & $A$ & $a d e$ & $\emptyset$ & $a d e$ & $a d e$ & $\emptyset$ & $\emptyset$ & $\emptyset$ & $\emptyset$ & $\emptyset$ & $\emptyset$ & $\emptyset$ \\
2 & $b c$ & $\emptyset$ & $a b c$ & $a c d e$ & $b c$ & $\emptyset$ & $a c d e$ & $\emptyset$ & $\emptyset$ & $\emptyset$ & $\emptyset$ & $\emptyset$ & $\emptyset$ & $\emptyset$ \\
4 & $b c$ & $b$ & $a b c$ & $\emptyset$ & $b c$ & $b$ & $\emptyset$ & $\emptyset$ & $\emptyset$ & $\emptyset$ & $\emptyset$ & $\emptyset$ & $\emptyset$ & $\emptyset$ \\
12 & $\emptyset$ & $\emptyset$ & $a b c$ & $a d e$ & $\emptyset$ & $\emptyset$ & $a d e$ & $\emptyset$ & $\emptyset$ & $\emptyset$ & $\emptyset$ & $\emptyset$ & $\emptyset$ & $\emptyset$ \\
13 & $\emptyset$ & $d e$ & $\emptyset$ & $d e$ & $\emptyset$ & $d e$ & $d e$ & $\emptyset$ & $\emptyset$ & $\emptyset$ & $\emptyset$ & $\emptyset$ & $\emptyset$ & $\emptyset$ \\
24 & $b c$ & $\emptyset$ & $a b c$ & $\emptyset$ & $b c$ & $\emptyset$ & $\emptyset$ & $\emptyset$ & $\emptyset$ & $\emptyset$ & $\emptyset$ & $\emptyset$ & $\emptyset$ & $\emptyset$ \\
123 & $\emptyset$ & $\emptyset$ & $\emptyset$ & $d e$ & $\emptyset$ & $\emptyset$ & $d e$ & $\emptyset$ & $\emptyset$ & $\emptyset$ & $\emptyset$ & $\emptyset$ & $\emptyset$ & $\emptyset$ \\
124 & $\emptyset$ & $\emptyset$ & $a b c$ & $\emptyset$ & $\emptyset$ & $\emptyset$ & $\emptyset$ & $\emptyset$ & $\emptyset$ & $\emptyset$ & $\emptyset$ & $\emptyset$ & $\emptyset$ & $\emptyset$ \\
$U$ & $\emptyset$ & $\emptyset$ & $\emptyset$ & $\emptyset$ & $\emptyset$ & $\emptyset$ & $\emptyset$ & $\emptyset$ & $\emptyset$ & $\emptyset$ & $\emptyset$ & $\emptyset$ & $\emptyset$ & $\emptyset$ \\
$\emptyset$ & $\emptyset$ & $\emptyset$ & $\emptyset$ & $\emptyset$ & $\emptyset$ & $\emptyset$ & $\emptyset$ & $\emptyset$ & $\emptyset$ & $\emptyset$ & $\emptyset$ & $\emptyset$ & $\emptyset$ & $\emptyset$ \\
\hline
\end{tabular}

Hence, for all $X_{i} \in L_{U}(U, A, \widetilde{I}), X_{j}=\left\{x_{j}\right\} \not \subset X_{i}$ such that $x_{j} \notin X_{i}^{*^{*}{ }^{*} D} \Rightarrow X_{i}=X_{i}^{*^{*}{ }^{*} D}$. So, there exists $\left(X_{i}, X_{i}^{*_{D}}\right) \in$ $L\left(U, D, \widetilde{I}_{D}\right)$. Thus, $D$ is a consistent set of $(U, A, \widetilde{I})$.

Definition 35. Let $(U, A, \widetilde{I})$ be an IF formal context and $\mathscr{D}^{*}$ be discernibility matrix of $(U, A, \widetilde{I})$. We define

$$
\begin{gathered}
\mathscr{M}^{*}=\underset{\mathscr{D}^{*}\left(X_{i}, X_{j}\right) \in \mathscr{D}^{*}}{\wedge}\left\{\underset{a_{k} \in \mathscr{D}^{*}\left(X_{i}, X_{j}\right)}{ } a_{k}\right\}, \\
\mathscr{D}^{*}\left(X_{i}, X_{j}\right) \neq \emptyset .
\end{gathered}
$$

Then, $\mathscr{M}^{*}$ is called discernibility function of the IF formal context $(U, A, \widetilde{I})$.

Proposition 36. Let $(U, A, \widetilde{I})$ be an IF formal context. The minimal disjunctive normal form of discernibility function is defined as

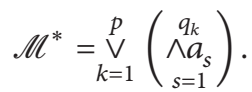

Denote $B_{k}=\left\{a_{s} \mid s=1,2, \ldots, q_{k}\right\}$, then $\left\{B_{k} \mid k=1,2, \ldots, p\right\}$ are all reductions of the IF formal context $(U, A, \widetilde{I})$.

Proof. It can be easily verified by Proposition 34, Definition 35, and the definition of minimal disjunctive normal of discernibility function.

From above discussion, we know that to get the attribute reductions in the IF concept lattices is equal to find the minimum consistent set $D$ which satisfies $D \cap \mathscr{D}^{*}\left(X_{i}, X_{j}\right) \neq \emptyset$ for any $\mathscr{D}^{*}\left(X_{i}, X_{j}\right) \neq \emptyset$.

Similarly, for any $X_{i} \in L_{U}(U, A, \widetilde{I})$ and $\mathscr{D}^{*}\left(X_{i}, X_{j}\right) \neq \emptyset$, the discernibility function of $X_{i}$ is defined as

$$
\mathscr{M}^{*}\left(X_{i}\right)=\wedge_{X_{j}=\left\{x_{j}\right\} \not \subset X_{i}}^{\wedge}\left\{\underset{a_{k} \in \mathscr{D}^{*}\left(X_{i}, X_{j}\right)}{\vee} a_{k}\right\}=\underset{k=1}{\vee}\left(\begin{array}{c}
q_{k} \\
\wedge a_{s} \\
s=1
\end{array}\right) .
$$

Corollary 37. $D$ is a consistent set of $(U, A, \widetilde{I}) \Leftrightarrow X^{*_{D}} \subseteq$ $X^{* *}$.

In the following, we investigate how to get IF attributes reductions from all attributes in concept lattices by illustrating an example.

Example 38 (renewal Example 22). All the reductions can be computed by discernibility matrix and discernibility function in Example 22.

By the definition of discernibility matrix, the results are presented in Table 2.

Hence, we can get that

$$
\begin{aligned}
\mathscr{M}^{*}= & (a \vee d \vee e) \wedge(a \vee b \vee c \vee d \vee e) \wedge(b \vee c) \\
& \wedge(a \vee b \vee c) \wedge(a \vee c \vee d \vee e) \wedge(d \vee e) \wedge b \\
= & b \wedge(d \vee e)=(b \wedge d) \vee(b \wedge e) .
\end{aligned}
$$

Through calculation and analysis, there are two reductions which are $D^{1}=\{b, d\}$ and $D^{2}=\{b, e\}$, for the IF formal context in Table 1 . The core attribute of the IF formal context is $b . d, e$ are relatively necessary attributes; $a, c$ are absolutely unnecessary attributes.

Algorithm 39. Discernibility functions are monotonic Boolean functions, and we obtain that the minimal disjunctive normal form of the discernibility function determine all the reductions. Algorithm of attribute reduction of concept based on the IF formal context is described as follows, and the flow chart of the algorithm is shown in Figure 3. Consider

Input: An IF formal context $K=(U, A, \widetilde{I})$, where $U=$ $\left\{x_{1}, x_{2}, \ldots, x_{n}\right\}, A=\left\{a_{1}, a_{2}, \ldots, a_{m}\right\}$.

Output: $\operatorname{RED}(K) / /$ All reductions of the IF formal context. 


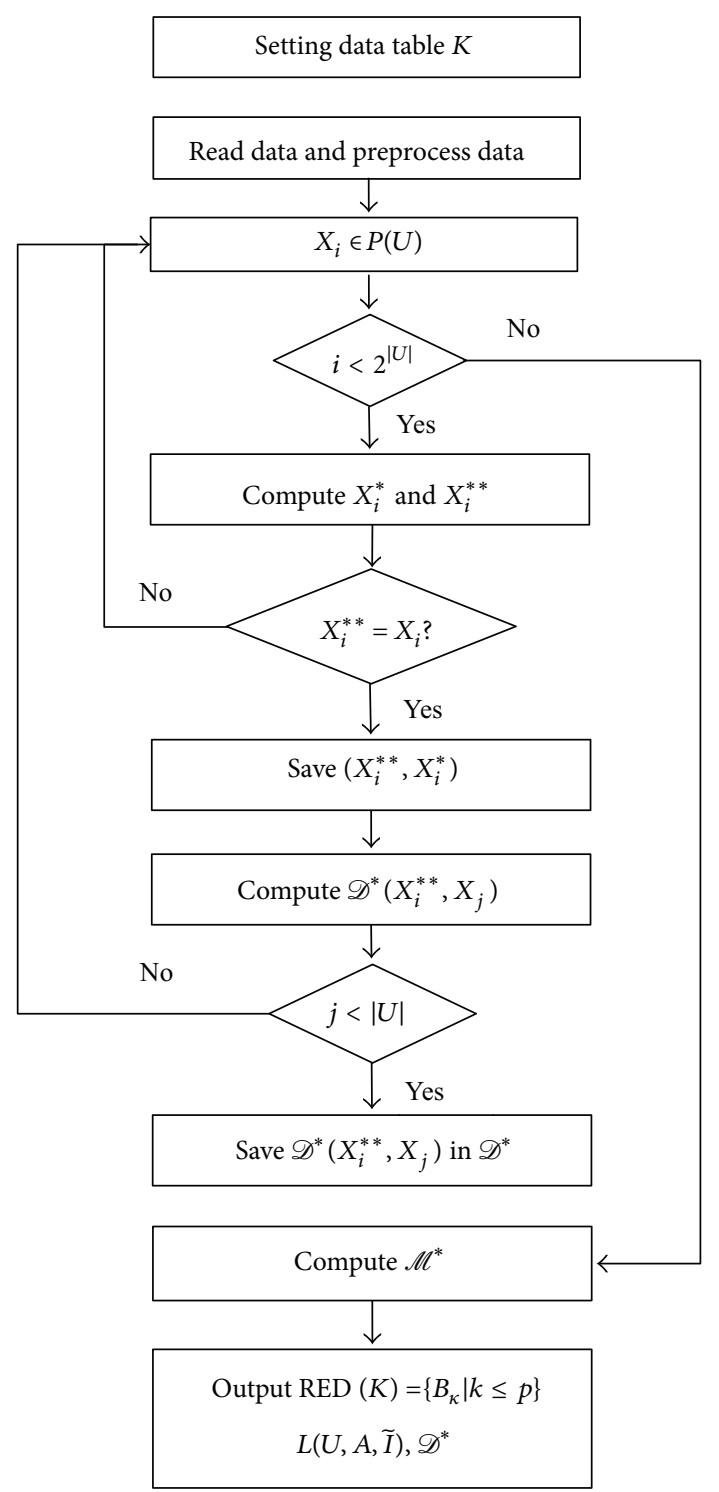

Figure 3: The flow chart of Algorithm 39.

Step 1: Initialized setting. We denote the initialized information by $K$ and read data table.

Step 2: Compute $X_{i}^{*}$ for $X_{i} \in P(U)$.

Step 3: Compute $X_{i}^{* *}$, the corresponding extension of $X_{i}^{*}$, according to Definitions 7 and 10 . And remove duplicates.

Step 4: Compute the set of discernibility attributes $\mathscr{D}^{*}\left(X_{i}^{* *}, X_{j}\right)$ for according to Definition 33 .

Step 5: Compute $\mathscr{M}^{*}=\wedge_{\mathscr{D}^{*}\left(X_{i}, X_{j}\right) \in \mathscr{D}^{*}}\left\{\vee\left\{a_{k} \mid a_{k} \in\right.\right.$ $\left.\left.\mathscr{D}^{*}\left(X_{i}^{* *}, X_{j}\right)\right\}\right\}$.

Step 6: Compute $\mathscr{M}^{*}=\mathrm{V}_{k=1}^{p}\left(\wedge a_{s s=1}^{q_{k}}\right)$.

Step 7: Let $B_{k}=\left\{a_{s} \mid s \leq q_{k}\right\}$ and $\operatorname{RED}(K)=\left\{B_{k} \mid k \leq p\right\}$.

Step 8: Output $L(U, A, \widetilde{I}), \mathscr{D}^{*}$ and $\operatorname{RED}(K)$.
TABLE 3: The target IF formal context $K$.

\begin{tabular}{cccccc}
\hline & $a$ & $b$ & $c$ & $d$ & $e$ \\
\hline$x_{1}$ & $\langle 0.9,0.0\rangle$ & $\langle 0.8,0.1\rangle$ & $\langle 0.2,0.8\rangle$ & $\langle 0.2,0.5\rangle$ & $\langle 0.9,0.0\rangle$ \\
$x_{2}$ & $\langle 0.8,0.0\rangle$ & $\langle 0.8,0.1\rangle$ & $\langle 0.8,0.1\rangle$ & $\langle 0.3,0.5\rangle$ & $\langle 0.0,0.8\rangle$ \\
$x_{3}$ & $\langle 0.0,0.8\rangle$ & $\langle 0.0,0.6\rangle$ & $\langle 0.1,0.9\rangle$ & $\langle 0.8,0.2\rangle$ & $\langle 0.0,0.8\rangle$ \\
$x_{4}$ & $\langle 0.7,0.2\rangle$ & $\langle 0.8,0.1\rangle$ & $\langle 0.7,0.1\rangle$ & $\langle 0.2,0.6\rangle$ & $\langle 0.0,0.8\rangle$ \\
$x_{5}$ & $\langle 0.0,0.0\rangle$ & $\langle 0.1,0.0\rangle$ & $\langle 0.0,0.0\rangle$ & $\langle 0.9,0.1\rangle$ & $\langle 0.0,0.8\rangle$ \\
$x_{6}$ & $\langle 0.6,0.4\rangle$ & $\langle 0.9,0.0\rangle$ & $\langle 0.0,0.9\rangle$ & $\langle 0.8,0.2\rangle$ & $\langle 0.0,0.8\rangle$ \\
$x_{7}$ & $\langle 0.9,0.1\rangle$ & $\langle 0.8,0.1\rangle$ & $\langle 0.2,0.8\rangle$ & $\langle 0.2,0.6\rangle$ & $\langle 0.0,0.8\rangle$ \\
\hline
\end{tabular}

In the worst case, the time complexity of the proposed algorithm is $O\left(2^{|U|}|U||A|\right)$, where $|U|$ is the number of objects and $|A|$ is the number of attributes. If the number of attributes has an upper bound, as usually happens, the cost of time grows geometrically with the number of objects.

Experimental computing program can be designed and carried out so as to apply the algorithm studied more directly and applicably. The main process of the program will be introduced by the flow chart. According to Algorithm 39, the process of the program can be designed and listed in Figure 3: the flow chart of the program.

Furthermore, the program has been employed to compute all concepts and reductions of the IF formal context in Example 8, which are consistent with the results obtained in Example 38. The test shows that the program is effective.

Example 40. Let $K=(U, A, \widetilde{I})$ be an IF formal context about some emerging viruses presented in Table 3, where $U=$ $\left\{x_{1}, x_{2}, x_{3}, x_{4}, x_{5}, x_{6}, x_{7}\right\}$ is the set of emerging viruses and $A=\left\{a_{1}, a_{2}, a_{3}, a_{4}, a_{5}\right\}$ is the set of some important characteristics about viruses. The interpretations of the attributes are listed as follows.

$\left(a_{1}\right)$ The type of genome's nucleic acids, where membership degree is referred to as DNA; nonmembership degree is referred to as RNA.

$\left(a_{2}\right)$ Envelope.

$\left(a_{3}\right)$ Strand: membership degree is referred to as single strand; nonmembership degree is referred to as double strand.

$\left(a_{4}\right)$ The heredity of protein.

$\left(a_{5}\right)$ Pathogenicity: membership degree is referred to as pathogenicit; nonmembership degree is referred to as opposite.

We can obtain one reduction $\operatorname{RED}(K)=\left\{a_{1}, a_{3}, a_{4}\right\}$ and thirty concepts of $K$, of which detailed description are shown in Table 4.

The results obtained above presented the relations between these new viruses which may be useful to find their ancestors and evolution mechanism for the viruses' researchers. In a way, the approach to construction and attribute reduction of IF concept lattice, presented in the paper, can be used to prune the redundancies and process the information. 
TABLE 4: The results of Example 40.

\begin{tabular}{|c|c|c|c|}
\hline Concept & Extension & Intension & Discernibility function of $X_{i}$ \\
\hline 1 & $x_{1}$ & $\{\langle 0.9,0.0\rangle,\langle 0.8,0.1\rangle,\langle 0.2,0.8\rangle,\langle 0.2,0.5\rangle,\langle 0.9,0.0\rangle\}$ & $a_{1} \vee a_{5}$ \\
\hline 2 & $x_{2}$ & $\{\langle 0.8,0.0\rangle,\langle 0.8,0.1\rangle,\langle 0.8,0.1\rangle,\langle 0.3,0.5\rangle,\langle 0.0,0.8\rangle\}$ & $\left(a_{1} \wedge a_{4}\right) \vee a_{3}$ \\
\hline 3 & $x_{3}$ & $\{\langle 0.0,0.8\rangle,\langle 0.0,0.6\rangle,\langle 0.1,0.9\rangle,\langle 0.8,0.2\rangle,\langle 0.0,0.8\rangle\}$ & $a_{3} \wedge a_{4}$ \\
\hline 4 & $x_{5}$ & $\{\langle 0.0,0.0\rangle,\langle 0.1,0.0\rangle,\langle 0.0,0.0\rangle,\langle 0.9,0.1\rangle,\langle 0.0,0.8\rangle\}$ & $\left(a_{1} \wedge a_{2}\right) \vee a_{3} \vee a_{4}$ \\
\hline 5 & $x_{6}$ & $\{\langle 0.6,0.4\rangle,\langle 0.9,0.0\rangle,\langle 0.0,0.9\rangle,\langle 0.8,0.2\rangle,\langle 0.0,0.8\rangle\}$ & $\left(a_{1} \wedge a_{4}\right) \vee a_{2}$ \\
\hline 6 & $x_{1}, x_{2}$ & $\{\langle 0.8,0.0\rangle,\langle 0.8,0.1\rangle,\langle 0.2,0.8\rangle,\langle 0.2,0.5\rangle,\langle 0.0,0.8\rangle\}$ & $a_{1} \vee\left(a_{3} \wedge a_{4}\right)$ \\
\hline 7 & $x_{1}, x_{7}$ & $\{\langle 0.9,0.1\rangle,\langle 0.8,0.1\rangle,\langle 0.2,0.8\rangle,\langle 0.2,0.6\rangle,\langle 0.0,0.8\rangle\}$ & $a_{1}$ \\
\hline 8 & $x_{2}, x_{3}$ & $\{\langle 0.0,0.8\rangle,\langle 0.0,0.6\rangle,\langle 0.1,0.9\rangle,\langle 0.3,0.5\rangle,\langle 0.0,0.8\rangle\}$ & $a_{3} \wedge a_{4}$ \\
\hline 9 & $x_{2}, x_{4}$ & $\{\langle 0.7,0.2\rangle,\langle 0.8,0.1\rangle,\langle 0.7,0.1\rangle,\langle 0.2,0.6\rangle,\langle 0.0,0.8\rangle\}$ & $a_{3}$ \\
\hline 10 & $x_{2}, x_{5}$ & $\{\langle 0.0,0.0\rangle,\langle 0.1,0.0\rangle,\langle 0.0,0.1\rangle,\langle 0.3,0.5\rangle,\langle 0.0,0.8\rangle\}$ & $\left(a_{1} \wedge a_{3}\right) \vee\left(a_{1} \wedge a_{4}\right) \vee\left(a_{3} \wedge a_{4}\right)$ \\
\hline 11 & $x_{2}, x_{6}$ & $\{\langle 0.6,0.4\rangle,\langle 0.8,0.1\rangle,\langle 0.0,0.9\rangle,\langle 0.3,0.5\rangle,\langle 0.0,0.8\rangle\}$ & $\left(a_{1} \wedge a_{4}\right) \vee\left(a_{2} \wedge a_{4}\right)$ \\
\hline 12 & $x_{5}, x_{6}$ & $\{\langle 0.0,0.4\rangle,\langle 0.1,0.0\rangle,\langle 0.0,0.9\rangle,\langle 0.8,0.2\rangle,\langle 0.0,0.8\rangle\}$ & $\left(a_{1} \wedge a_{4}\right) \vee a_{2}$ \\
\hline 13 & $x_{1}, x_{2}, x_{3}$ & $\{\langle 0.0,0.8\rangle,\langle 0.0,0.6\rangle,\langle 0.1,0.9\rangle,\langle 0.2,0.5\rangle,\langle 0.0,0.8\rangle\}$ & $a_{3} \wedge a_{4}$ \\
\hline 14 & $x_{1}, x_{2}, x_{5}$ & $\{\langle 0.0,0.0\rangle,\langle 0.1,0.1\rangle,\langle 0.0,0.8\rangle,\langle 0.2,0.5\rangle,\langle 0.0,0.8\rangle\}$ & $a_{1} \vee\left(a_{3}, a_{4}\right)$ \\
\hline 15 & $x_{1}, x_{2}, x_{6}$ & $\{\langle 0.6,0.4\rangle,\langle 0.8,0.1\rangle,\langle 0.0,0.9\rangle,\langle 0.2,0.5\rangle,\langle 0.0,0.8\rangle\}$ & $\left(a_{1} \wedge a_{4}\right) \vee\left(a_{2} \wedge a_{4}\right)$ \\
\hline 16 & $x_{1}, x_{2}, x_{7}$ & $\{\langle 0.8,0.1\rangle,\langle 0.8,0.1\rangle,\langle 0.2,0.8\rangle,\langle 0.2,0.6\rangle,\langle 0.0,0.8\rangle\}$ & $a_{1}$ \\
\hline 17 & $x_{2}, x_{4}, x_{5}$ & $\{\langle 0.0,0.2\rangle,\langle 0.1,0.1\rangle,\langle 0.0,0.1\rangle,\langle 0.2,0.6\rangle,\langle 0.0,0.8\rangle\}$ & $a_{3}$ \\
\hline 18 & $x_{2}, x_{5}, x_{6}$ & $\{\langle 0.0,0.4\rangle,\langle 0.1,0.0\rangle,\langle 0.0,0.9\rangle,\langle 0.3,0.5\rangle,\langle 0.0,0.8\rangle\}$ & $\left(a_{1} \wedge a_{4}\right) \vee\left(a_{2} \wedge a_{4}\right)$ \\
\hline 19 & $x_{3}, x_{5}, x_{6}$ & $\{\langle 0.0,0.8\rangle,\langle 0.0,0.6\rangle,\langle 0.0,0.9\rangle,\langle 0.8,0.2\rangle,\langle 0.0,0.8\rangle\}$ & $a_{4}$ \\
\hline 20 & $x_{1}, x_{2}, x_{4}, x_{7}$ & $\{\langle 0.7,0.2\rangle,\langle 0.8,0.1\rangle,\langle 0.2,0.8\rangle,\langle 0.2,0.6\rangle,\langle 0.0,0.8\rangle\}$ & $a_{1} \vee a_{3}$ \\
\hline 21 & $x_{1}, x_{2}, x_{5}, x_{6}$ & $\{\langle 0.0,0.4\rangle,\langle 0.1,0.1\rangle,\langle 0.0,0.9\rangle,\langle 0.2,0.5\rangle,\langle 0.0,0.8\rangle\}$ & $\left(a_{1} \wedge a_{4}\right) \vee\left(a_{2} \wedge a_{4}\right)$ \\
\hline 22 & $x_{1}, x_{2}, x_{5}, x_{7}$ & $\{\langle 0.0,0.1\rangle,\langle 0.1,0.1\rangle,\langle 0.0,0.8\rangle,\langle 0.2,0.6\rangle,\langle 0.0,0.8\rangle\}$ & $a_{1}$ \\
\hline 23 & $x_{2}, x_{3}, x_{5}, x_{6}$ & $\{\langle 0.0,0.8\rangle,\langle 0.0,0.6\rangle,\langle 0.0,0.9\rangle,\langle 0.3,0.5\rangle,\langle 0.0,0.8\rangle\}$ & $a_{4}$ \\
\hline 24 & $x_{1}, x_{2}, x_{3}, x_{4}, x_{7}$ & $\{\langle 0.0,0.8\rangle,\langle 0.0,0.6\rangle,\langle 0.1,0.9\rangle,\langle 0.2,0.6\rangle,\langle 0.0,0.8\rangle\}$ & $a_{3}$ \\
\hline 25 & $x_{1}, x_{2}, x_{3}, x_{5}, x_{6}$ & $\{\langle 0.0,0.8\rangle,\langle 0.0,0.6\rangle,\langle 0.0,0.9\rangle,\langle 0.2,0.5\rangle,\langle 0.0,0.8\rangle\}$ & $a_{4}$ \\
\hline 26 & $x_{1}, x_{2}, x_{4}, x_{5}, x_{7}$ & $\{\langle 0.0,0.2\rangle,\langle 0.1,0.1\rangle,\langle 0.0,0.8\rangle,\langle 0.2,0.6\rangle,\langle 0.0,0.8\rangle\}$ & $a_{1} \vee a_{3}$ \\
\hline 27 & $x_{1}, x_{2}, x_{4}, x_{6}, x_{7}$ & $\{\langle 0.6,0.4\rangle,\langle 0.8,0.1\rangle,\langle 0.0,0.9\rangle,\langle 0.2,0.6\rangle,\langle 0.0,0.8\rangle\}$ & $a_{1} \vee a_{2}$ \\
\hline 28 & $x_{1}, x_{2}, x_{4}, x_{5}, x_{6}, x_{7}$ & $\{\langle 0.0,0.4\rangle,\langle 0.1,0.1\rangle,\langle 0.0,0.9\rangle,\langle 0.2,0.6\rangle,\langle 0.0,0.8\rangle\}$ & $a_{1} \vee a_{2}$ \\
\hline 29 & $U$ & $\{\langle 0.0,0.8\rangle,\langle 0.0,0.6\rangle,\langle 0.0,0.9\rangle,\langle 0.2,0.6\rangle,\langle 0.0,0.8\rangle\}$ & - \\
\hline 30 & $\emptyset$ & $\{\langle 1.0,0.0\rangle,\langle 1.0,0.0\rangle,\langle 1.0,0.0\rangle,\langle 1.0,0.0\rangle,\langle 1.0,0.0\rangle\}$ & $\vee_{i=1}^{7} a_{i}$ \\
\hline $\operatorname{RED}(K)$ & & $\left\{a_{1}, a_{3}, a_{4}\right\}$ & \\
\hline
\end{tabular}

\section{Conclusion}

This paper introduced the intuitionistic fuzzy set into the concept lattice theory firstly. Then, we offered the related definitions and propositions of attribute reduction in concept lattices which were examined by some examples. What is more, the discernibility matrix and discernibility function in concept lattice were defined which presented an approach to attribute reduction of concept lattice based on the intuitionistic fuzzy context. The attribute reduction of IF concept lattice made the representation of implicit knowledge simpler in IF formal context. In fact, an approach to reduction was introduced based on the discernibility matrix of IF concept lattice. The results of this paper extended the theory of concept lattice, although there were some problems in the applications.

\section{Acknowledgments}

This work is supported by the National Natural Science Foundation of China (no. 61105041), Science and Technology Program of Board of Education of Chongqing (no. KJ120805), National Natural Science Foundation of CQ
CSTC (no. cstc2013jcyjA40051), and Postgraduate Innovation Foundation of Chongqing University of Technology (no. YCX2012203).

\section{References}

[1] R. Wille, "Restructuring lattice theory: an approach based on hierarchies of concepts," in Ordered Sets, vol. 83, pp. 445-470, Reidel, Dordrecht, The Netherlands, 1982.

[2] K. Y. Hu, Y. C. Lu, and C. Y. Shi, "Advances in concept lattice and its application," Journal of Tsinghua University, vol. 40, no. 9, pp. $77-81,2000$

[3] G. D. Oosthuizen, "The application of concept lattice to machine learning," Tech. Rep., University of Pretoria, Pretoria, South Africa, 1996.

[4] W. H. Xu, J. Z. Pang, and S. Q. Luo, "Novel cognitive system model and approach to transformation of information granules," International Journal of Approximate Reasoning, 2013.

[5] K. T. Atanassov, "Intuitionistic fuzzy sets," Fuzzy Sets and Systems, vol. 20, no. 1, pp. 87-96, 1986.

[6] K. T. Atanassov, Intuitionistic Fuzzy Sets, Physica, Heidelberg, Germany, 1999. 
[7] L. A. Zadeh, "Fuzzy sets," Information and Computation, vol. 8, pp. 338-353, 1965.

[8] G. Deschrijver and E. E. Kerre, "On the position of intuitionistic fuzzy set theory in the framework of theories modelling imprecision," Information Sciences, vol. 177, no. 8, pp. 1860-1866, 2007.

[9] L. Lin, X.-H. Yuan, and Z.-Q. Xia, "Multicriteria fuzzy decisionmaking methods based on intuitionistic fuzzy sets," Journal of Computer and System Sciences, vol. 73, no. 1, pp. 84-88, 2007.

[10] I. K. Vlachos and G. D. Sergiadis, "Intuitionistic fuzzy information-applications to pattern recognition," Pattern Recognition Letters, vol. 28, no. 2, pp. 197-206, 2007.

[11] Z. S. Xu, "Intuitionistic preference relations and their application in group decision making," Information Sciences, vol. 177, no. 11, pp. 2363-2379, 2007.

[12] C. Carpineto and G. Romano, "GALOIS: an order-theoretic approach to conceptual clustering," in Proceedings of 10th International Conference on Machine Learning, P. Utgo, Ed., vol. 293, pp. 33-40, Elsevier, Amherst, Mass, USA, 1993.

[13] I. Düntsch and G. Gediga, "Algebraic aspects of attribute dependencies in information systems," Fundamenta Informaticae, vol. 29, no. 1-2, pp. 119-133, 1997.

[14] R. Godin, R. Missaoui, and H. Alaoui, "Incremental concept formation algorithms based on Galois (concept) lattices," Computational Intelligence, vol. 11, no. 2, pp. 246-267, 1995.

[15] J. Pócs, "Note on generating fuzzy concept lattices via Galois connections," Information Sciences, vol. 185, pp. 128-136, 2012.

[16] J. H. Li and Y. J. Lü, "Attribute reduction and rules extraction in decision formal context based on concept lattice," Mathematics in Practice and Theory, vol. 39, no. 7, pp. 182-188, 2009.

[17] J. H. Li, C. L. Mei, and Y. J. Lv, "Incomplete decision contexts: approximate concept construction, rule acquisition and knowledge reduction," International Journal of Approximate Reasoning, vol. 54, no. 1, pp. 149-165, 2013.

[18] G. D. Oosthuizen, "Rough sets and concept lattices," in Rough Sets, and IF Sets and Knowledge Discovery (RSKD '93), W. P. Ziarko, Ed., pp. 24-31, Springer, London, UK, 1994.

[19] Z. Pawlak, "Rough sets," International Journal of Computer and Information Sciences, vol. 11, no. 5, pp. 341-356, 1982.

[20] Z. Pawlak and A. Skowron, "Rudiments of rough sets," Information Sciences, vol. 177, no. 1, pp. 3-27, 2007.

[21] J. B. Zhang, T. R. Li, D. Ruan, and D. Liu, "Rough sets based matrix approaches with dynamic attribute variation in set-valued information systems," International Journal of Approximate Reasoning, vol. 53, no. 4, pp. 620-635, 2012.

[22] R. E. Kent, "Rough concept analysis: a synthesis of rough sets and formal concept analysis," Fundamenta Informaticae, vol. 27, no. 2-3, pp. 169-181, 1996.

[23] L. Wei, Rough Theory and Concept Approach, Xian Jiaotong University, Xi'an, China, 2005.

[24] X. J. Zhu, T. Cheng, and H. Q. Yang, "The attribute reduction in intuitiontics fuzzy concept lattice," Journal of Hebei Normal University (Nature Science), vol. 35, pp. 119-123, 2011.

[25] C. Aswani Kumar and S. Srinivas, "Concept lattice reduction using fuzzy K-Means clustering," Expert Systems with Applications, vol. 37, no. 3, pp. 2696-2704, 2010.

[26] R. Bělohlávek and J. Macko, "Selecting important concepts using weights," in Proceedings of the 9th International Conference on Formal Concept Analysis, P. Valtchev and R. Jäschke, Eds., vol. 6628 of Lecture Notes in Computer Science, pp. 65-80, 2011.
[27] B. Ganter and R. Wille, Formal Concept Analysis, Springer, Berlin, Germany, 1999.

[28] L. Li and J. Zhang, "Attribute reduction in fuzzy concept lattices based on the T implication," Knowledge-Based Systems, vol. 23, no. 6, pp. 497-503, 2010.

[29] J. H. Li, C. L. Mei, and Y. J. Lv, "Knowledge reduction in real decision formal contexts," Information Sciences, vol. 189, pp. 191207, 2012.

[30] J. H. Li, C. L. Mei, Y. J. Lv, and X. Zhang, "A heuristic knowledge reduction algorithm for real decision formal contexts," in Rough Sets and Current Trends in Computing, vol. 7413 of Lecture Notes in Artificial Intelligence, pp. 303-312, 2012.

[31] J. H. Li, C. L. Mei, and Y. J. Lv, "Knowledge reduction in formal decision contexts based on an order-preserving mapping," International Journal of General Systems, vol. 41, no. 2, pp. 143$161,2012$.

[32] M. Liu, M. W. Shao, W. X. Zhang, and C. Wu, "Reduction method for concept lattices based on rough set theory and its application," Computers \& Mathematics with Applications, vol. 53, no. 9, pp. 1390-1410, 2007.

[33] D. Pei and J.-S. Mi, "Attribute reduction in decision formal context based on homomorphism," International Journal of Machine Learning and Cybernetics, vol. 2, no. 4, pp. 289-293, 2011.

[34] D. Pei, M.-Z. Li, and J.-S. Mi, "Attribute reduction in fuzzy decision formal contexts," in Proceedings of the International Conference on Machine Learning and Cybernetics (ICMLC '11), pp. 204-208, July 2011.

[35] H. Wang and W.-X. Zhang, "Approaches to knowledge reduction in generalized consistent decision formal context," Mathematical and Computer Modelling, vol. 48, no. 11-12, pp. 1677$1684,2008$.

[36] X. Wang, "Approaches to attribute reduction in concept lattices based on rough set theory," International Journal of Hybrid Information Technology, vol. 5, pp. 67-80, 2012.

[37] W.-Z. Wu, Y. Leung, and J.-S. Mi, "Granular computing and knowledge reduction in formal contexts," IEEE Transactions on Knowledge and Data Engineering, vol. 21, no. 10, pp. 1461-1474, 2009.

[38] Y. Y. Yao, Y. Zhao, and J. Wang, "On reduct construction algorithms," in Proceedings of the 1st International Conference on Rough Sets and Knowledge Technology, vol. 4062 of Lecture Notes in Artificial Intelligence, pp. 297-304, 2006.

[39] W. X. Zhang, L. Wei, and J. J. Qi, "Attribute reduction theory and approach to concept lattice," Science in China F, vol. 48, no. 6, pp. 713-726, 2005.

[40] W. X. Zhang, L. Wei, and J. J. Qi, "Attribute reduction in concept lattice based on discernibility matrix," in Rough Sets, Fuzzy Sets, Data Mining, and Granular Computing, D. Slezak, J. Yao, J. F. Peters, W. Ziarko, and H. Xiaohua, Eds., vol. 642 of Lecture Notes in Artificial Intelligence, pp. 157-165, 2005.

[41] J. Outrata and V. Vychodil, "Fast algorithm for computing fixpoints of Galois connections induced by object-attribute relational data," Information Sciences, vol. 185, pp. 114-127, 2012. 


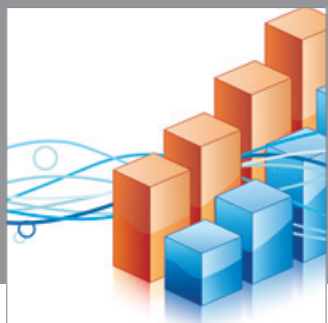

Advances in

Operations Research

mansans

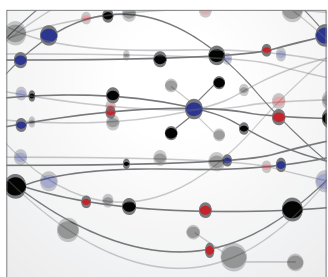

The Scientific World Journal
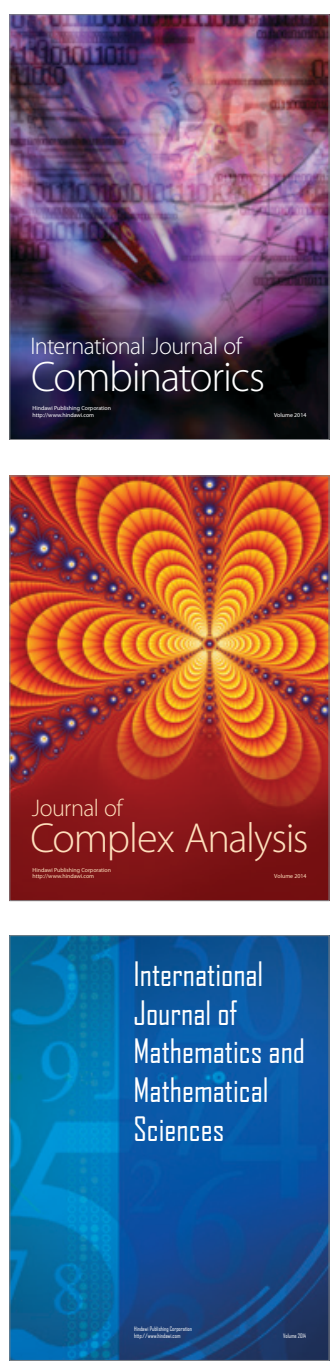
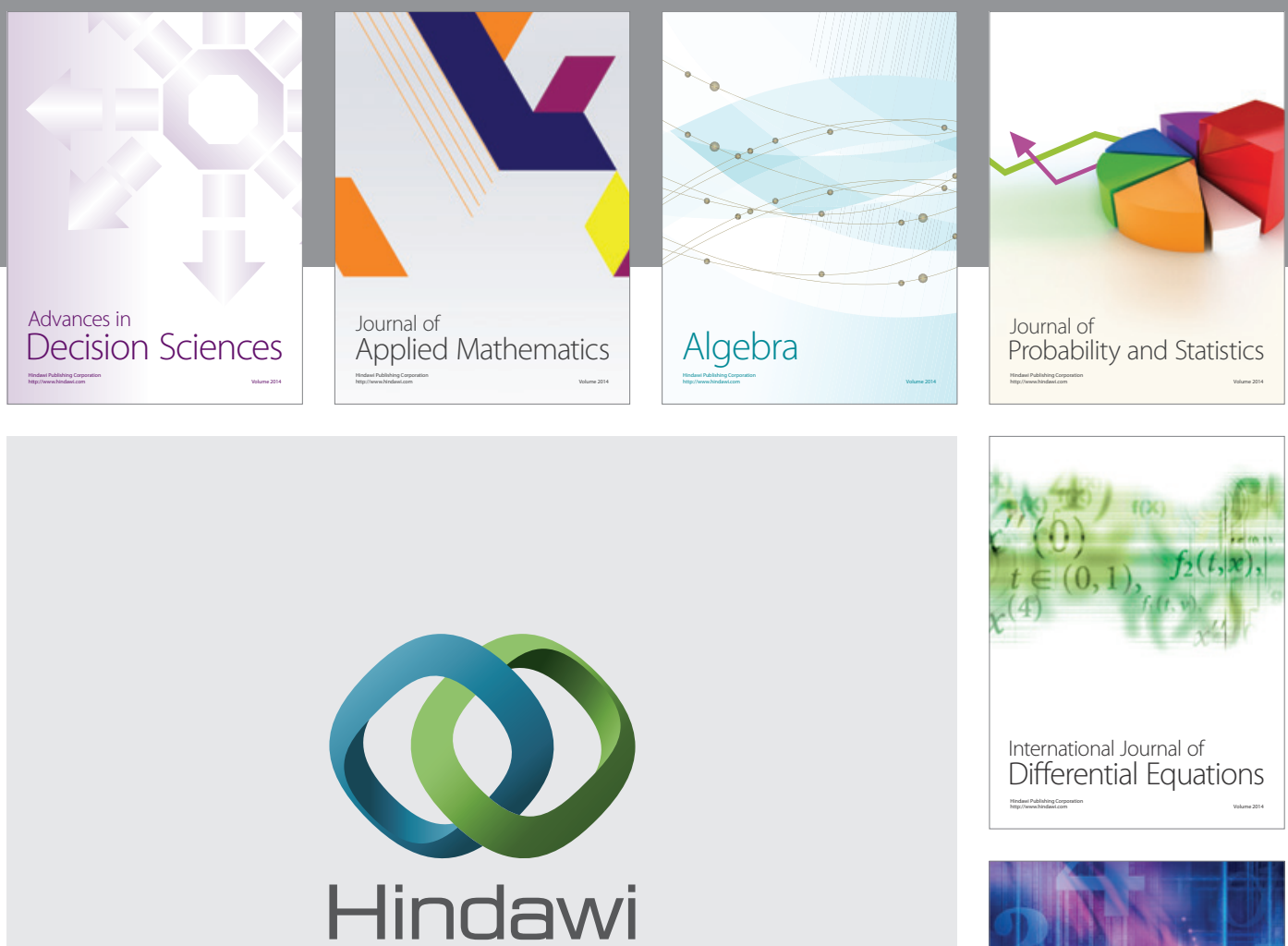

Submit your manuscripts at http://www.hindawi.com
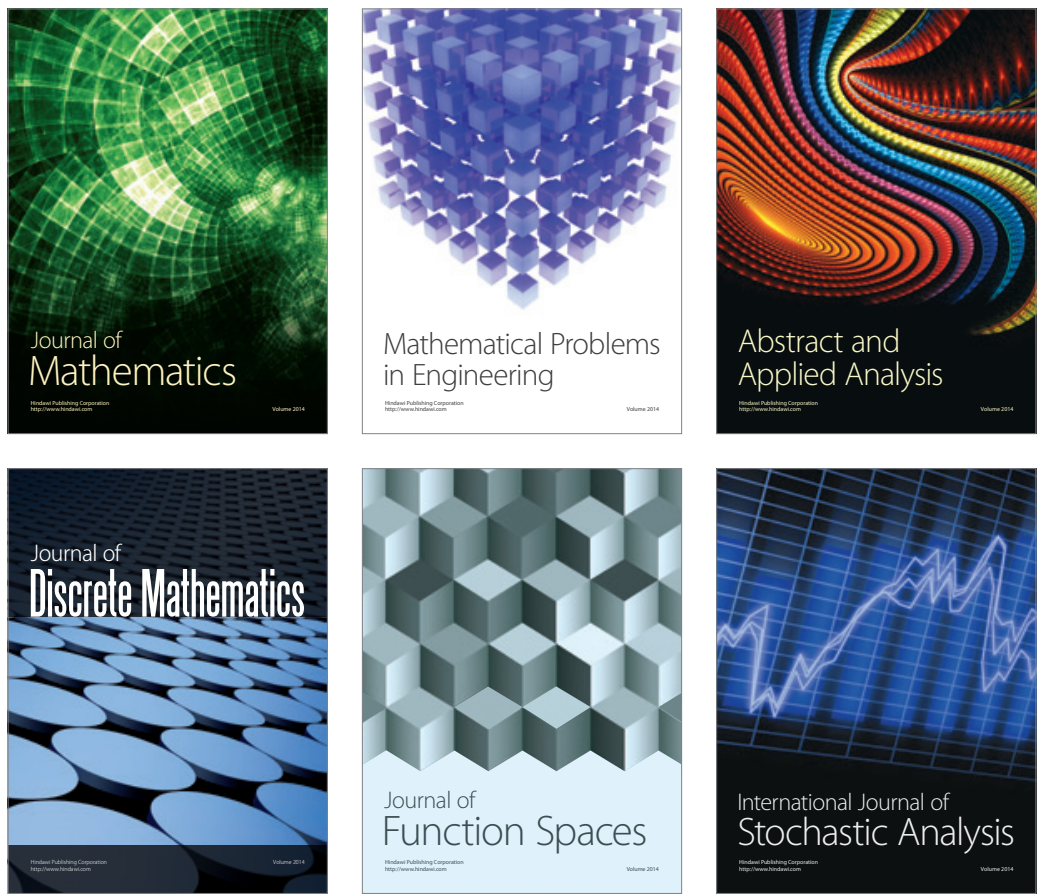

Journal of

Function Spaces

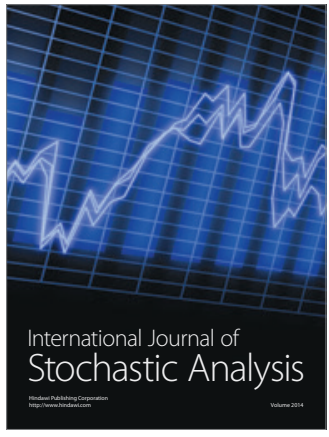

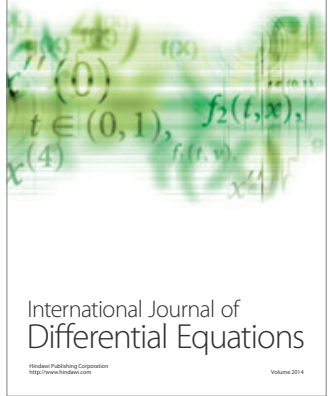
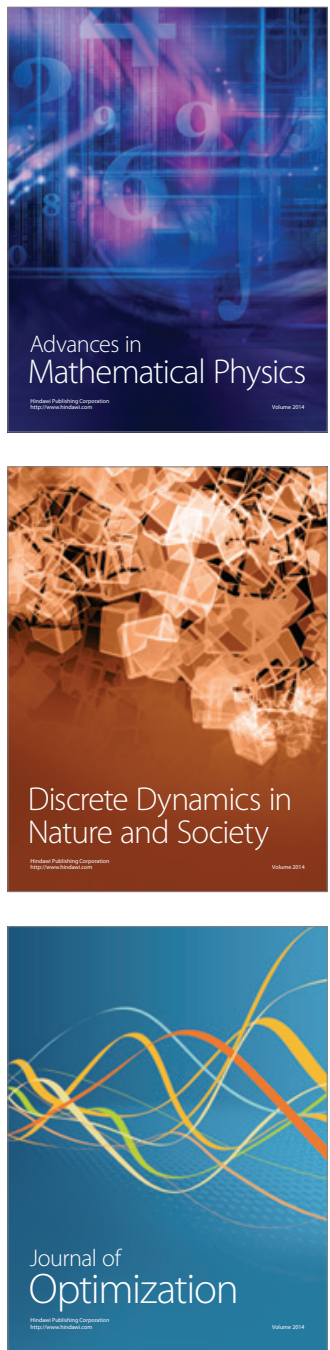